\title{
Un consolidamento antico con inzeppature metalliche in un paramento lapideo a Iasos (Caria)
}

\section{An ancient stone facing strengthening with metal wedges at Iasos (Caria)}

\author{
Paolo Vitti ${ }^{1}$ \\ Università di Roma Tre
}

\begin{abstract}
RIASSUNTO
L'articolo analizza un consolidamento antico su una parete delle grandi terme a Iasos di Caria. L'analisi si basa sull'osservazione dell'impiego inconsueto di elementi di ferro, inseriti in abbondanza nei giunti di una parete del caldarium. Il rilievo e lo studio delle caratteristiche costruttive del muro hanno condotto all'identificazione di un dissesto del paramento. Quest'ultimo, formato da grandi e pesanti blocchi appena sbozzati e non ammorsati al nucleo della muratura in conglomerato, si distaccò forse per effetto di un terremoto. L'impiego di zeppe di ferro per creare contrasto tra le pietre di un paramento dissestato, costituiva una soluzione ottimale per consolidare la muratura senza dover smontare I'intero paramento.
\end{abstract}

Parole chiave: consolidamento; cunei e zeppe di ferro; muratura in conglomerato; Iasos; terme romane; caldarium.

\begin{abstract}
This paper discusses a masonry strengthening on the wall of the Great bath building at Iasos in Caria. The analysis is based on the observation of a peculiar use of iron elements, inserted in the joints of a wall of the caldarium. The survey and the study of the wall has demonstrated that the facing of the wall separated from its core, probably due to an earthquake. The damage is likely to have occurred because of the big and heavy stones of the facing which were not bonded to the core of the wall. The use of iron wedges might have been an ideal solution for strengthening the wall without dismantling it.
\end{abstract}

Key words: strengthening; iron wedges; rubble masonry; Iasos; Roman baths; caldarium.

\section{RESUMEN}

El artículo analiza una consolidación antigua en una pared de la gran terma en Iasos de Caria. El análisis se basa en la observación del uso inusual de elementos de hierro, insertados abundantemente en las juntas de una pared del caldarium. La inspección y estudio de las características constructivas del muro permitieron identificar un deterioro del revestimiento. Este último, formado por grandes y pesados bloques apenas esbozados y no unidos al núcleo del muro, se desprendió quizás por efecto de un terremoto. El uso de la cuña de hierro para crear contraste entre las piedras de un paramento constituía una solución óptima para consolidar la mampostería sin tener que desmontar todo el revestimiento.

Palabras clave: consolidación; cuñas y zapatas de hierro; mampostería en conglomerado; Iasos; termas romanas; caldarium.

Recibido: 26-01-2017. Aceptado: 24-09-2017. Publicado online: 12-12-2017

\section{Cómo citar este artículo / Citation}

Vitti, P. 2017: "Un consolidamento antico con inzeppature metalliche in un paramento lapideo a Iasos (Caria)", Arqueología de la Arquitectura, 14: e061, doi: http://dx.doi.org/10.3989/arq.arqt.2017.009.

\section{Copyright}

(c) 2017 CSIC. Este es un artículo de acceso abierto distribuido bajo los términos de una licencia de uso y distribución Creative Commons Attribution (CC-by) España 3.0. 


\section{INTRODUZIONE}

Nel 2008 la Missione Archeologica Italiana di Iasos ha avviato le indagini nel "castello di Terraferma" di Iasos di Caria (moderna Kıyıkışlacık) sotto la direzione di Alessandro Viscogliosi, di Sapienza Università di Roma $^{2}$. Si tratta di un'area chi si trova a Nord dell'agorà, nel punto in cui il promontorio occupato dall'insediamento antico si salda alla Terraferma (Fig. 1). La cinta muraria, che si è formata per addizioni successive in un arco cronologico ampio $^{3}$, recinge una serie di edifici in

2 Viscogliosi 2009 e 2010.

3 Viscogliosi 2010: 19. rovina, ad oggi indagati solo nella parte emergente dal piano di campagna. Il settore orientale del "castello di Terraferma" è occupato da un grande edificio termale (Fig. 2), la cui datazione, in mancanza di scavi archeologici, rimane incerta. Il complesso è tuttavia più antico rispetto al settore orientale della fortificazione, come si evince dal fatto che la costruzione delle mura ha qui inglobato l'abside di uno degli ambienti delle terme (Fig. 2: BT).

Una sintetica descrizione dell'edificio termale è stata pubblicata nel $2010^{4}$. L'ingresso era collocato sul

4 Ibidem, 23-24. Una pubblicazione esaustiva del "castello di Terraferma" è in corso di preparazione.

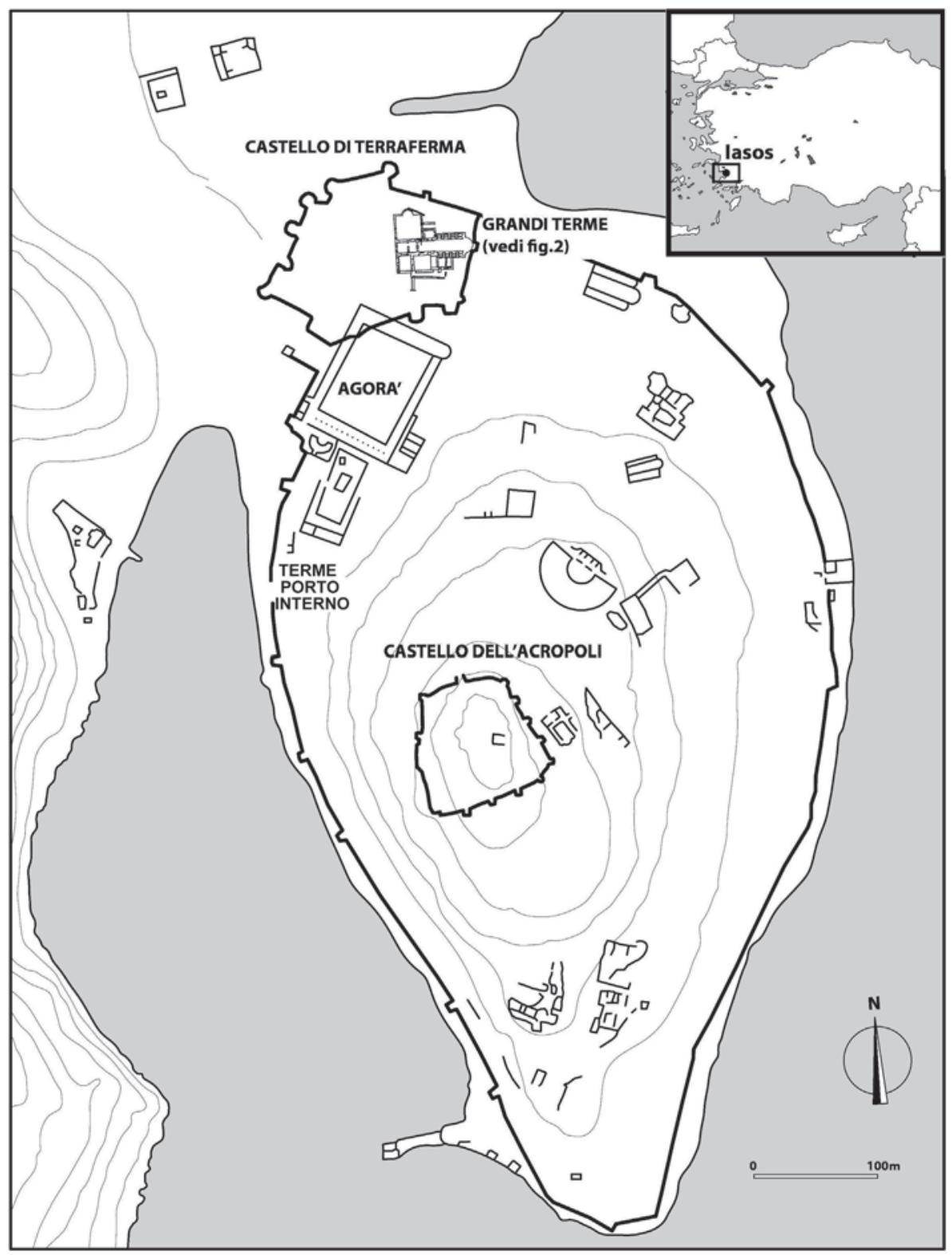

Fig. 1: Pianta di Iasos con localizzazione dell'impianto termale all'interno del "castello di Terraferma" (P. Vitti da Iasos di Caria). 


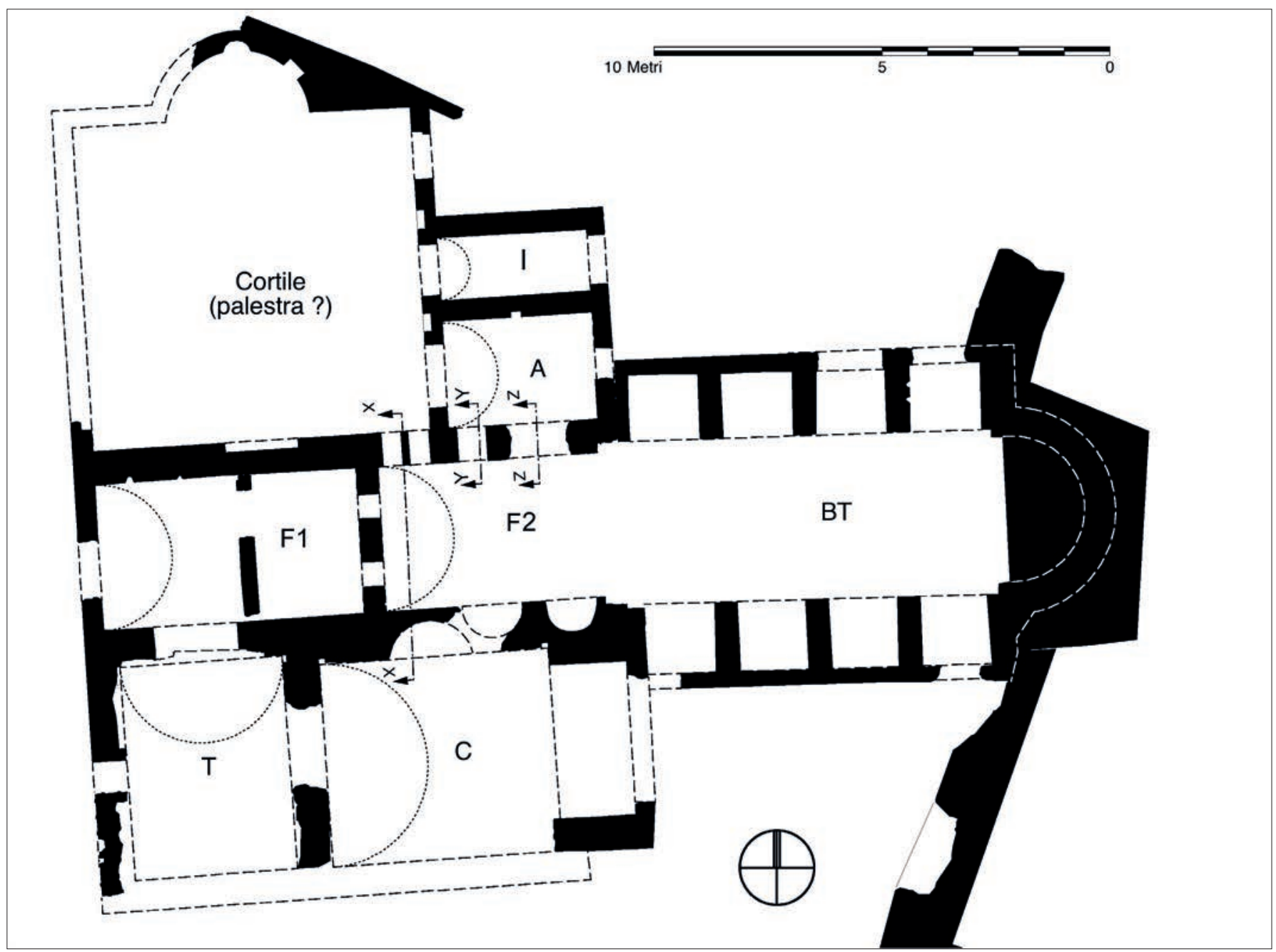

Fig. 2: Pianta delle grandi terme di Iasos (P. Vitti da elaborazione A. La Paglia).

lato rivolto verso l'insenatura collocata a Nord-Est del promontorio (Fig. 1). Da un vestibolo (Fig. 2: I) si entrava in un cortile/palestra di forma quadrata, con un ninfeo semicircolare sul lato Nord. A fianco al vestibolo, aperto sul cortile, era un ambiente identificato come apodyterium (Fig. 2: A). Il frigidarium, composto da due unità (Fig. 2: F1 e F2), si interpone tra il cortile e gli ambienti termali caldi (Fig. 2: T e C) collocati a Sud e una basilica termarum (Fig. 2: BT), se tale può essere l'identificazione di questa sala absidata con alcove laterali, aggiunta in un secondo momento ad Est.

Sulle pareti si riconoscono le tracce di rivestimenti marmorei, ancorati alle pareti lapidee con grappe metalliche infisse. Tuttavia, sulla parete Nord del caldarium (Fig. 3) gli elementi metallici in ferro sono numerosissimi e conficcati uno accanto all'altro nei giunti del paramento (Fig. 4). Nel testo che segue si proporrà una interpretazione di questa singolare evidenza sulla base di una analisi critica della parete in cui sono conficcati gli elementi metallici ${ }^{5}$.

\footnotetext{
5 Il rilievo delle terme è stato condotto da un gruppo di studenti della Facoltà di Architettura di Sapienza Università di Roma con il coordinamento di Nicolò Masturzo e Alessandro Viscogliosi. La mia analisi grafica si è basata sui rilievi rielaborati da Alessio La Paglia, che ringrazio, in vista della sua tesi di laurea. La finalità del mio lavoro era la comprensione dell'impiego dei ferro nei giunti dalle pietre. A tal fine, con base la restituzione al cad del rilievo ortofotografico, ho eseguito un disegno diretto della parete, così da entrare nella logica del processo costruttivo, addentrandomi nella relazione che lega i paramenti ai nuclei murari. Un lavoro di osservazioni maturate attraverso un esercizio grafico, che ha come base la necessità di comprendere le specificità della struttura, la natura dei materiali, la loro lavorazione, il loro assemblaggio, possibili difetti nella soluzione adottata, per individuare le cause di un dissesto e, conseguentemente, la necessità di un intervento di consolidamento. Nella valutazione critica della realtà è stata quindi data priorità a questioni di tecnica costruttiva, di comportamento strutturale delle masse in condizioni di esercizio statico e dinamico, di carichi e distribuzione delle masse. Il lettore tenga presente che il rilievo è stato condotto in scala 1:50, con incertezze fino a $10 \mathrm{~cm}$, pari a $2 \mathrm{~mm}$ nel disegno in scala. Un lavoro più puntuale sarà oggetto di pubblicazione in un volume dedicato al castello di Terraferma, a cura di Alessandro Viscogliosi.
} 


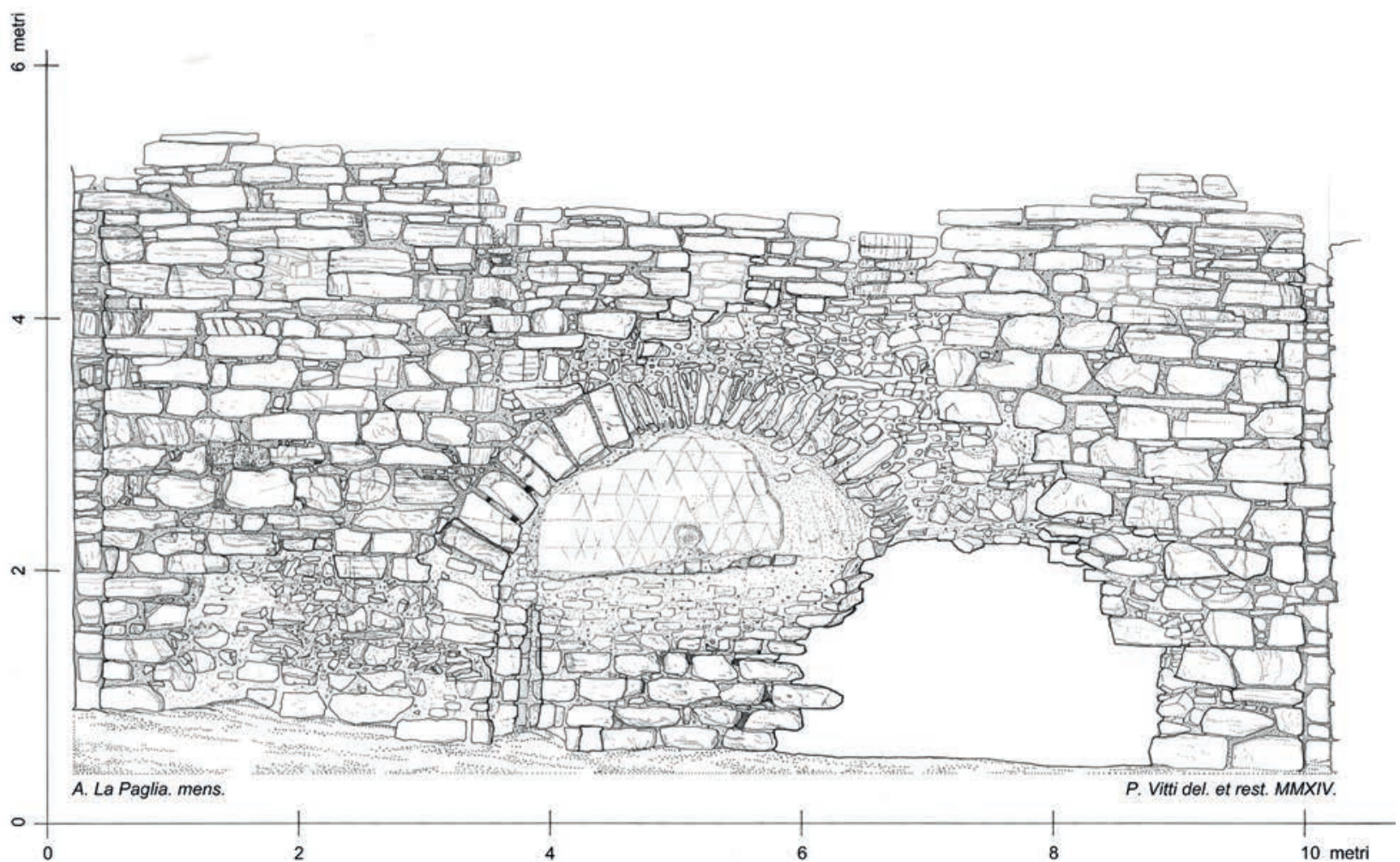

Fig. 3: Rilievo della parete Nord dell'ambiente (C). (P. Vitti da elaborazione A. La Paglia).

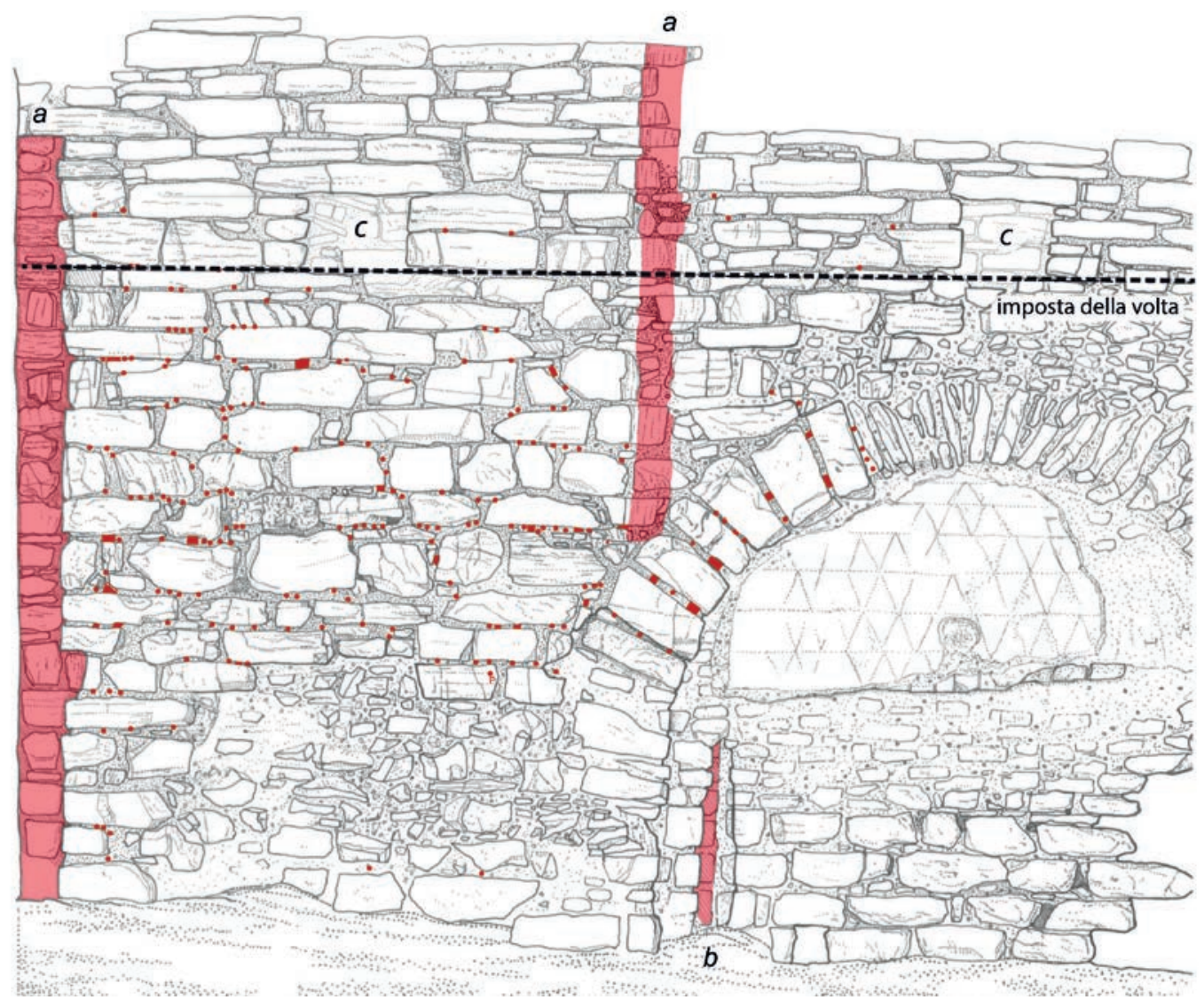

Fig. 4: Settore orientale della parete in Fig. 3 con indicazione degli elementi in ferro infissi tra le pietre. a) sfiatatoi nella parete per l'evacuazione dei fumi nell'ipocausto; b) incavo nella conca per l'evacuazione dei fumi dell'ipocausto; c) rientranze rettangolari sopra all'imposta della volta per l'inserimento delle travi che sorreggevano la centina. 


\section{DESCRIZIONE DELLA PARETE CON ELEMENTI METALLICI}

Il caldarium (C) è un ambiente rettangolare di ca. 10x8,60 m (Fig. 2: C), del quale sopravvivono sole tre dei quattro lati che lo definivano (Ovest, Est, Nord). La parete Est presenta una grande nicchia rettangolare $(6,70 \times 3,60 \mathrm{~m} \mathrm{ca}$. $)$ in cui poteva essere ubicato un alveus (vasca calda per l'immersione).

La parete Nord, dove sono impiegati gli elementi metallici, è quella meglio conservata, anche se la parte inferiore del paramento si è distaccata ed è caduta, lasciando a vista il nucleo in conglomerato. Verso gli angoli con le pareti Ovest ed Est vi sono due incavi, 26 x $26 \mathrm{~cm}$, che corrono a piena altezza. In essi erano collocati i tubuli attraverso i quali evacuavano i fumi provenienti dall'ipocausto (Fig. 4). L'incavo sulla sinistra è aperto verso l'ambiente fino a $70 \mathrm{~cm}$ sopra alla quota di imposta della volta (Fig. 5). Al di sopra di questa quota esso proseguiva verticalmente nella volta. Altri due incavi, di eguale funzione, erano collocati verso il centro della parete, e si innestavano al di sopra di una grande nicchia centrale.

La nicchia, ampia ca. 3,60 m, forse ospitava un labrum (bacino per le abluzioni). La ghiera in conci di ca. $40 \mathrm{~cm}$ di altezza è conservata solo sulla parte sinistra (Fig. 6). Nella parte mancante si osserva la tessitura della volta della nicchia, composta da scaglie di pietra di forma allungata, che formano la superficie emisferica. Le scaglie sono inserite in abbondante malta.

Sul catino sono evidenti le impronte lasciate da una decorazione contraddistinta da elementi triangolari accostati a formare un disegno geometrico per fasce orizzontali (Fig. 3), la cui appartenenza alla fase originale è dubbia. Una medesima decorazione, meglio conservata, ornava l'abside del ninfeo sul lato Nord del cortile.

In alto, la volta a botte si conserva per una altezza di ca. $100 \mathrm{~cm}$. L'imposta della volta (Fig. 4, linea tratteggiata orizzontale) è collocata ca. un metro sopra alla sommità della nicchia. L'intradosso della volta è in perfetta continuità con il piano del muro sottostante e si distingue da esso sia per la leggera curvatura, sia per la diversa forma e dimensione delle pietre; queste hanno una altezza minore, una lunghezza maggiore, presentano una forma rettangolare allungata e sono disposte per filari continui orizzontali. Sulla linea di imposta sono tre incassi rettangolari (Fig. 3 e Fig. 4: c), quello di

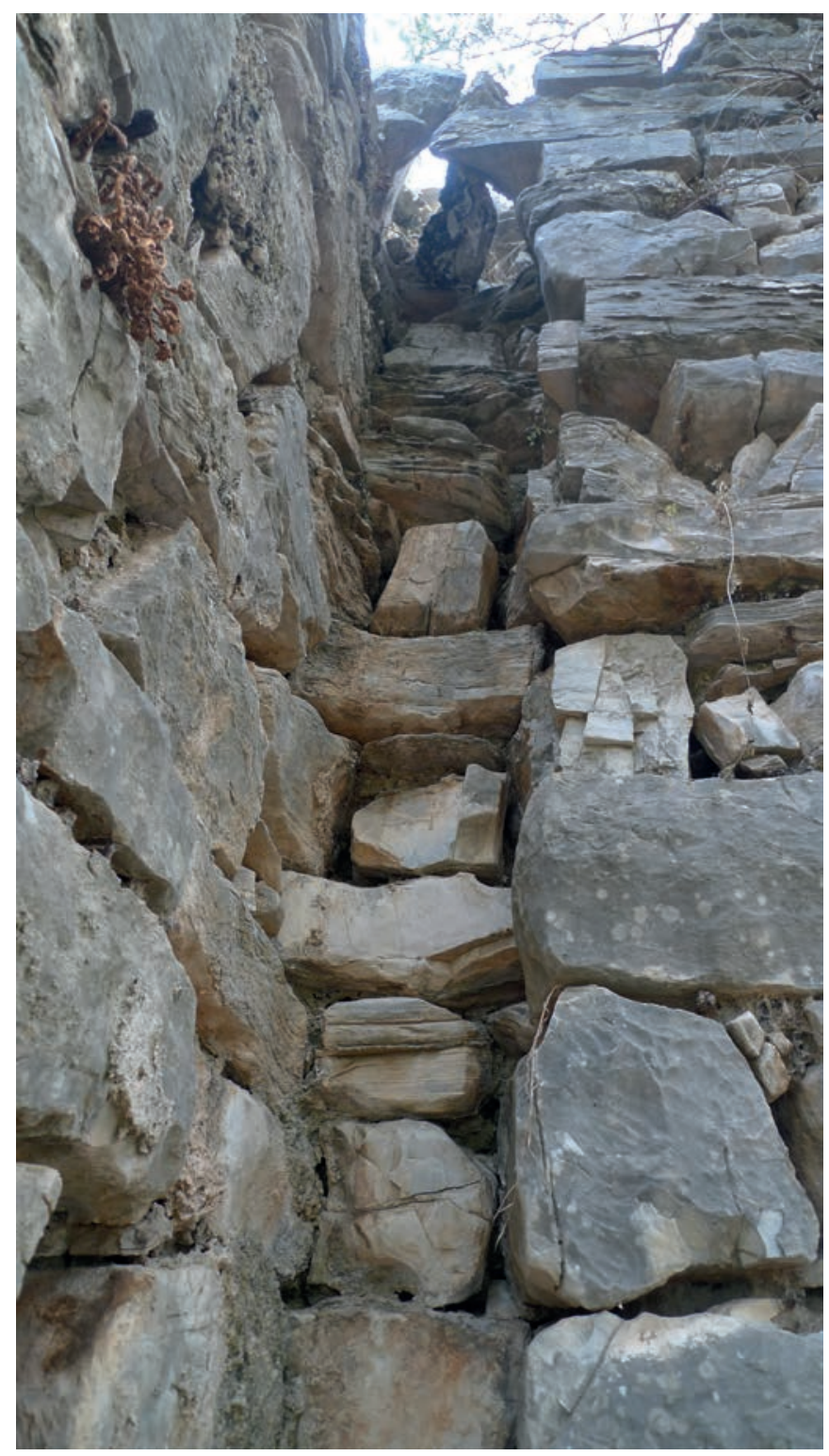

Fig. 5: Particolare dell'incasso in cui correva il condotto di evacuazione dei fumi dell'ipocausto. In alto il condotto proseguiva verticalmente nella volta.

sinistra misura $50 \mathrm{~cm}$ di ampiezza, $38 \mathrm{~cm}$ di altezza ed è profondo $37 \mathrm{~cm}$. La lastra che lo copre è lesionata al centro, per effetto del peso della muratura sovrastante. La profondità del foro corrisponde allo spessore medio del paramento in pietra, come si osserva nel foro centrale, dove il paramento è parzialmente caduto (Fig. 7). Gli incassi alloggiavano le travi di legno che sostenevano la centina (cd. centina volante), che pertanto non era poggiata a terra per mezzo di una armatura. 


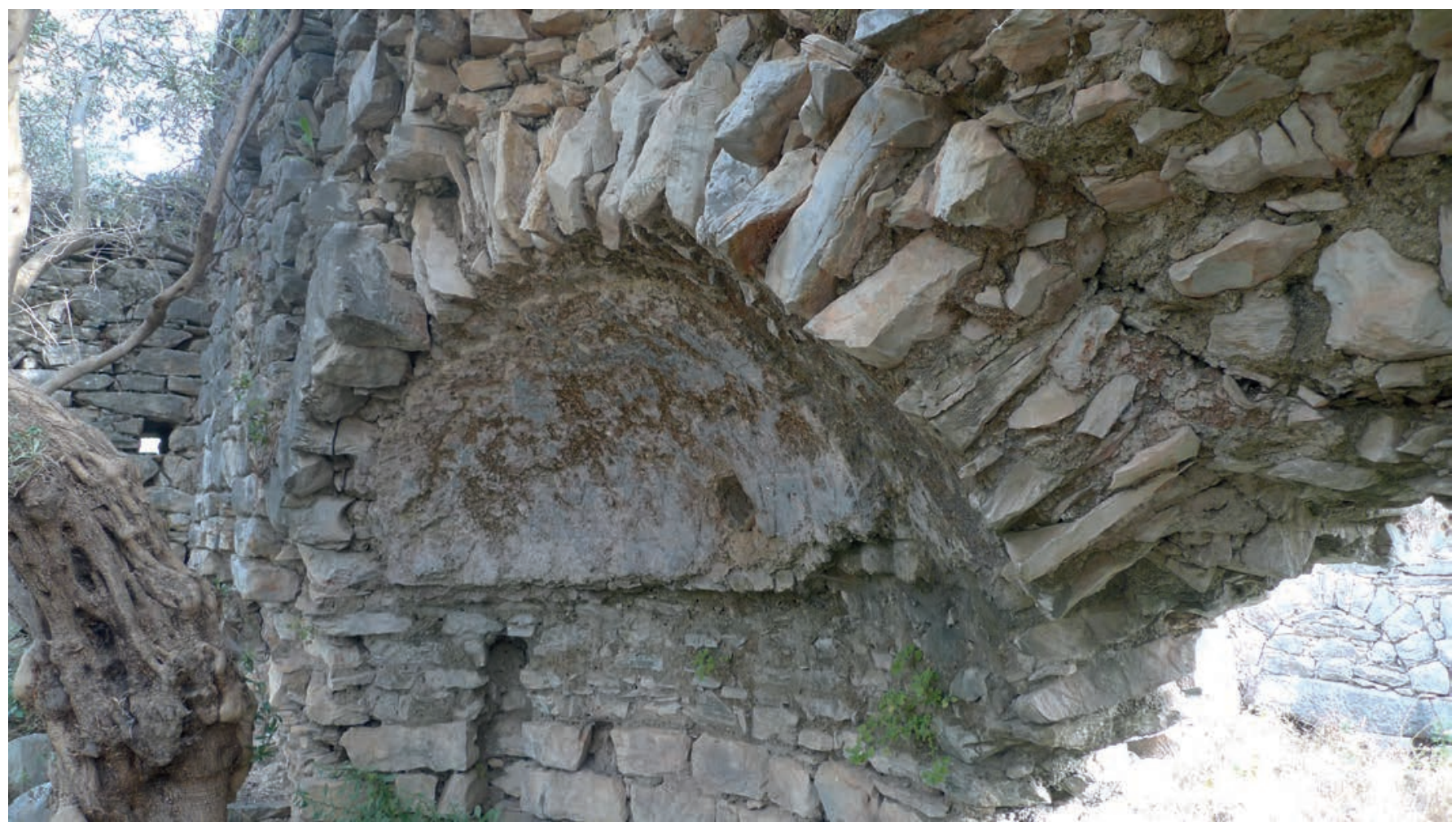

Fig. 6: Veduta di scorcio della tessitura della volta della calotta della nicchia.

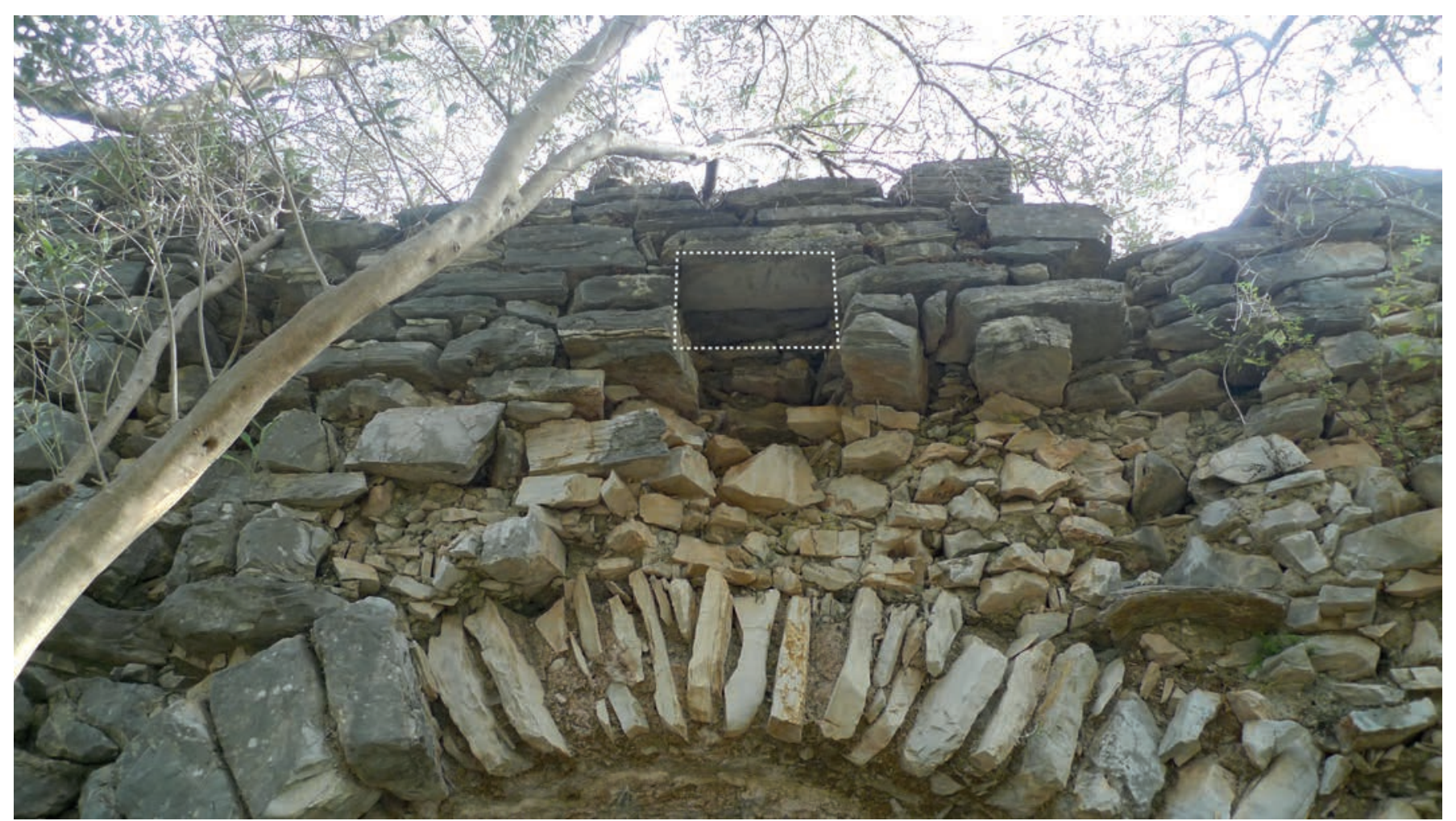

Fig. 7: Veduta dal basso della parte centrale della nicchia, dove appare evidente la differenza tra la dimensione dei blocchi dell'arco di facciata e delle pietre che formano il catino della nicchia. In tratteggio la sede di una delle travi che sorreggevano la centina della volta. 


\section{GLI ELEMENTI METALLICI}

Gli elementi metallici in ferro sono conficcati nei giunti del paramento in pietra (Fig. 4). Tracce di ossido sui letti di posa e di attesa dei blocchi del paramento giacenti a terra indicano che gli elementi metallici erano numerosi anche nella parte inferiore della parete, che è crollata. Viceversa, nella volta la loro presenza è piuttosto rada.

Per la quantità degli elementi metallici presenti sul paramento, la disposizione e distribuzione, si rimanda al disegno di dettaglio del settore occidentale della parete (Fig. 4). Essi sono generalmente circondati da malta, indubbiamente posta successivamente al loro inserimento, a rifinire l'intervento di inserimento del metallo nei giunti (Fig. 8). Quando la malta è assente, essi risultano in parte ossidati, e sono pertanto più sottili di quanto dovevano essere in antico. La loro lunghezza non eccede mai la profondità delle pietre, per cui non raggiungono mai il nucleo della muratura. Si distinguono alcuni elementi, con dimensioni variabili, di sezione quadrata $(2 \times 2,3 \times 3 \mathrm{~cm}$ ecc.) o rettangolare $(4 \times 2,6 \times 4 \mathrm{~cm}$, ecc.). Due elementi metallici più grandi, dei veri e propri cunei, sono collocati nella ghiera dell'arco di facciata della nicchia, nei giunti tra un concio e l'altro (Fig. 9); essi misurano $8 \times 3 \mathrm{~cm}$ e $8 \mathrm{x} 4 \mathrm{~cm}$. Altri cunei più piccoli sono presenti in altri punti della ghiera.

Di norma, in età romana, elementi metallici in forma di chiodi e grappe applicati su paramenti lapidei o laterizi potevano rendere più solidale l'intonaco al muro, ovvero servivano ad ancorare delle intercapedini addossate alle pareti ${ }^{6} \mathrm{o}$, infine, erano utilizzati per vincolare ai muri i rivestimenti lapidei o marmorei. Tuttavia, diverse osservazioni indicano che la parete di Iasos non ricade in alcuna delle sopra citate casistiche.

Non si tratta di chiodi per ancorare l'intonaco perché questi generalmente hanno una disposizione rada e non fitta come quelli che qui analizziamo.

Più plausibile sembra, in principio, l'utilizzo di questi elementi metallici per fissare tubuli rettangolari o tegulae mammate, così da formare una intercapedine riscaldata collegata all'ipocausto. Tale intercapedine è infatti indiziata dalla presenza di sfiatatoi per i fumi provenienti dall'ipocausto, formati da quattro incavi equamente distanziati sulla parete (Fig. 4: a), due alle estremità, che corrono a tutta altezza, e due sopra alla nicchia, che pertanto non sono direttamente collegati

\footnotetext{
6 Yegül 1992: 363-365.
}

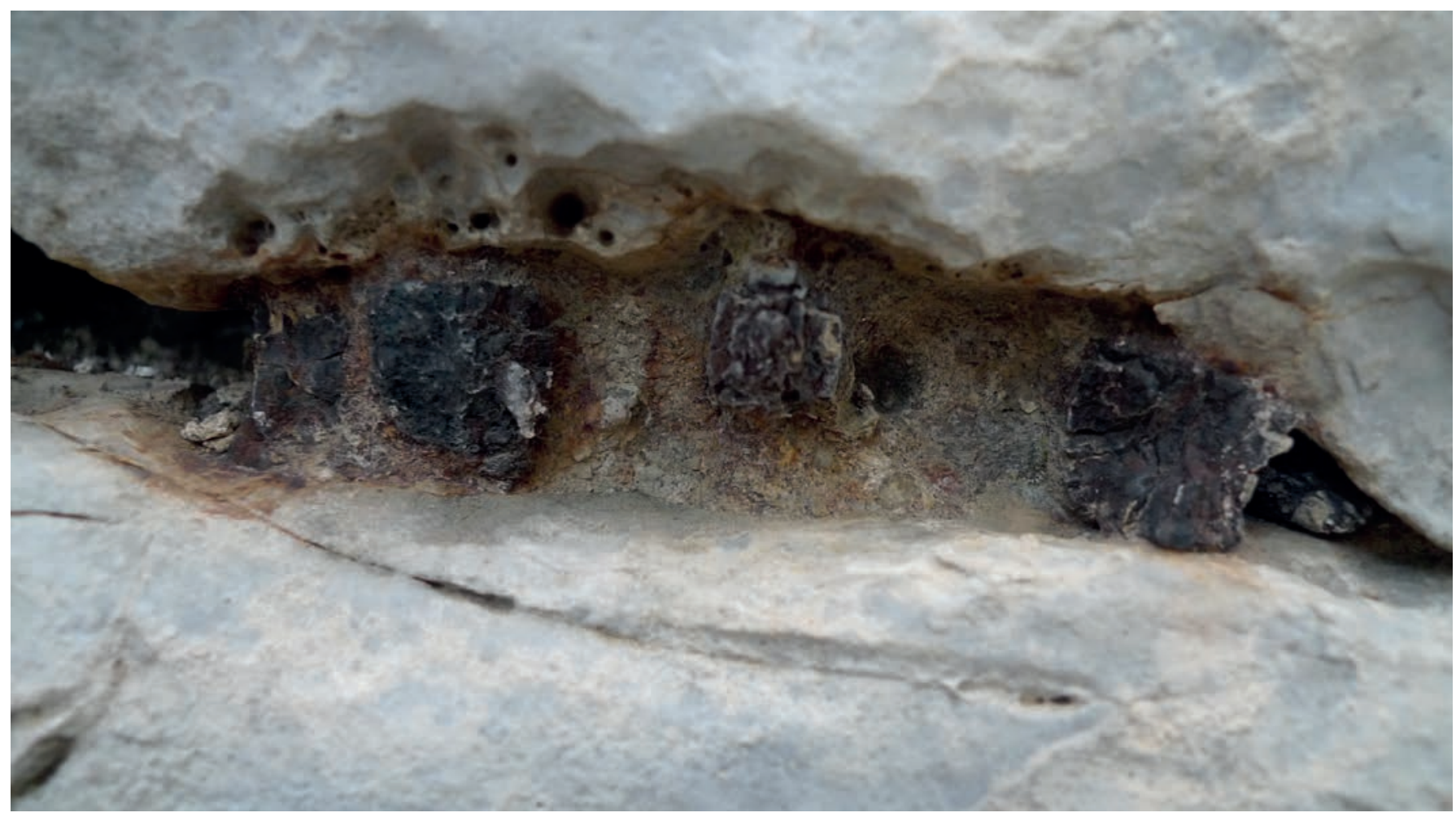

Fig. 8: Particolare di alcuni elementi metallici tra due pietre, circondati da malta aggiunta dopo il loro inserimento 


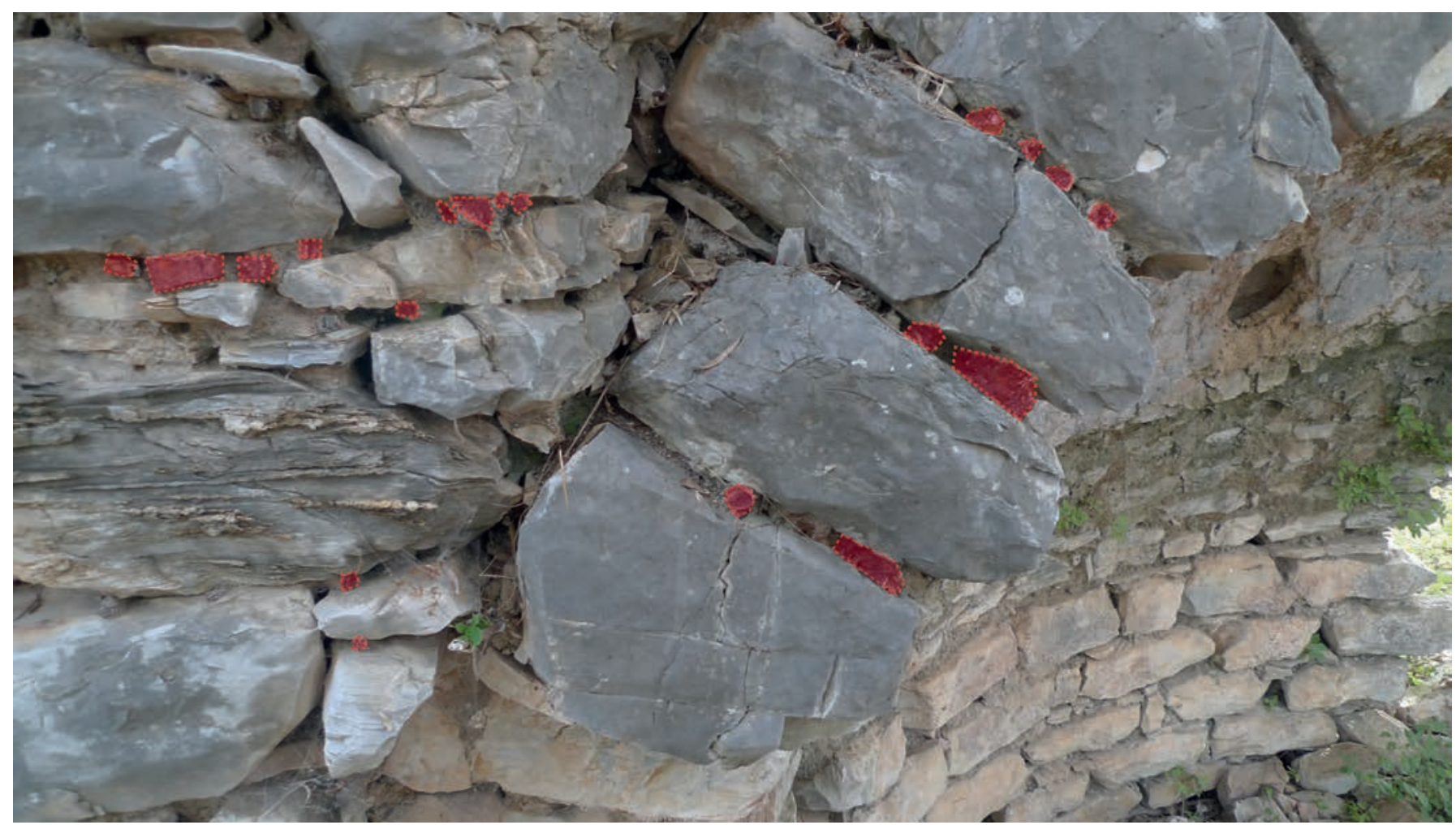

Fig. 9: Particolare dell'arco frontale della nicchia e della muratura, con evidenziazione degli elementi metallici tra le pietre.

all'ipocausto. Negli incavi dovevano essere collocati tubuli circolari in cui si incanalavano i fumi, come in altre terme antiche. Due tubuli circolari sono in effetti ancora presenti nella muratura interna della grande nicchia centrale (Fig. 4: b) e servivano alla esalazione dei gas provenienti dall'ipocausto nella parte bassa della parete centrale ${ }^{7}$. Tuttavia, ad una visione più attenta, è evidente che la quantità di chiodi è tale da non poter essere riconducibile al fissaggio dell'intercapedine. Infatti i chiodi utilizzati a tale scopo hanno una distribuzione piuttosto regolare e seguono la griglia geometrica dei tubuli o delle tegole da fissare. Nel nostro caso, invece, si nota che i chiodi sono disposti uno fianco all'altro, senza una regolare distribuzione e spesso in maniera molto fitta (Fig. 8).

Ugualmente, l'ipotesi che si tratti di grappe per l'ancoraggio di un rivestimento marmoreo ${ }^{8}$ va scartata, in quanto la parete in questione era con ogni probabilità rivestita da una intercapedine riscaldata e, pertanto, se mai grappe di questo tipo fossero esistite, esse avrebbero dovuto collocarsi sulla contro-parete addossata

\footnotetext{
7 Infatti i due incavi centrali nella parte alta della parete ricevevano i fumi solo dall'intercapedine.

8 Giuliani 2006: 189-191.
}

all'intercapedine e comunque avere una disposizione più rada e regolare. Inoltre, mentre negli altri ambienti dell'edificio i chiodi utilizzati per i rivesti marmorei, erano ancorati anche sulle pietre del paramento, nelle quali era ricavata la sede entro la quale fissare la grappa, nel caldarium (C) tutti gli elementi metallici, tranne uno, sono esclusivamente collocati nei giunti fra una pietra e l'altra.

La disposizione dei chiodi, così fitta e irregolare, ricorda in parte la chiodatura dei fogli di piombo sulle pareti che affacciano sull'atrio tuscanico della domus del fauno a Pompei, una soluzione tecnica ancora oggi poco chiara ${ }^{9}$, ma che comunque si contraddistingue per la compresenza di chiodi di ferro e lastre di piombo. Tuttavia nella nostra parete non vi è alcuna traccia di piombo.

L'impressione che l'inserimento degli elementi metallici sia dovuto ad un consolidamento del paramento dissestato sembra probabilmente la più plausibile. Per sostanziare tale ipotesi è opportuna una analisi della costruzione.

\footnotetext{
V. Hoffmann 2009
} 


\section{OSSERVAZIONI SU MURI E VOLTE}

\section{Le murature}

Lo spessore delle murature delle terme è variabile. Nel caldarium (C) e tepidarium (T) oscilla tra $150-160 \mathrm{~cm}$, mentre negli altri ambienti varia tra ca. 50 e $90 \mathrm{~cm}$. Gli spessori maggiori sembrano dipendere dal maggiore carico e dalle spinte generate dalle ampie volte in pietra degli ambienti riscaldati. Nell'ambiente BT, coperto anch'esso da un'ampia volta, gli spessori murari rimangono contenuti, grazie allo schema planimetrico con alcove, in cui i setti murari che risultano ortogonali all'ambiente centrale ricevono le spinte dalla volta centrale e le trasmettono alla base dei muri ${ }^{10}$. Fa eccezione la parete Nord del caldarium (C), dove lo spessore murario raggiunge ca. $210 \mathrm{~cm}$ così da inglobare la grande nicchia centrale, profonda $140 \mathrm{~cm}$. Infatti, la nicchia, con ogni probabilità destinata ad alloggiare il labrum, non fu creata in aggetto rispetto alla scatola muraria dell'ambiente, come è consuetudine in larga parte degli impianti termali romani, ma fu integrata nello spessore murario, così da non invadere l'ambiente F2. È questa una caratteristica precipua degli impianti termali microasiatici, già a suo tempo sottolineata da $\mathrm{F}$. Yegü $1^{11}$.

In Asia Minore, infatti, l'impianto planimetrico delle terme tende a schemi di aggregazione per cellule rettangolari, che lasciano poca possibilità a compenetrazioni spaziali. Differenza sostanziale, questa, rispetto agli schemi planimetrici che a Roma avevano condotto a quella sintesi compositiva e spaziale così originale. Non deve sfuggire che una simile soluzione era anche condizionata dalle tecniche costruttive: muri che facevano uso sporadico dell'ottimo cementizio di Roma e pesanti volte di pietra, che trasmettevano forti carichi ai piedritti, trovavano nei forti spessori delle murature una opportuna resistenza, soprattutto in un territorio non estraneo all'azione sismica.

Di fatto l'intera costruzione è realizzata integralmente in pietra, con la sola eccezione, per quanto osservabile dalle strutture sopra al piano di campagna, dei tubuli utilizzati per l'evacuazione dei fumi. D'altra parte il materiale da costruzione per eccellenza a Iasos è

\footnotetext{
10 Impianto che a Roma gode di una forte e illustre tradizione che si fa risalire a Traiano e alla creazione di ambienti rettangolari coperti da volte a crociera e fiancheggiati da ambienti più bassi ortogonali alla sala centrale e coperti da volte a botte (ad. esempio la grande aula dei Mercati di Traiano; v. Bianchini e Vitti 2017).

11 Yegül 1992: 251-254.
}

proprio la pietra. Nelle grandi terme il materiale lapideo appare sempre sbozzato sommariamente, minimamente lavorato e, nei nuclei, esso è lasciato a "spacco di cava" 12 (Fig. 10). La tecnica costruttiva utilizzata per formare le strutture portanti rientra nella categoria delle murature tripartite, con due paramenti ed un nucleo.

Il paramento è composto da pietre di $800-1100 \mathrm{~cm}^{2}$ di superficie, disposte per corsi. Le pietre, di forma irregolare e dimensione variabile, hanno la faccia a vista spianata sommariamente, quanto necessario per garantire la verticalità del paramento (Fig. 10), mentre le altre facce sono appena sbozzate. Le pietre sono disposte per corsi sub-orizzontali, vale a dire non sempre disposti lungo uniformi piani orizzontali, e il piano di posa è spesso regolarizzato con elementi lapidei di minore dimensione e scaglie disposti orizzontalmente, di dimensione e disposizione variabile. I giunti fra le grandi pietre sono vistosamente privi di malta (Fig. 11), tranne alcune "isole" attorno ai chiodi della parte Nord del caldarium (C). Le pietre, in origine, erano legate da malta, come si osserva in altre parti delle terme, dove, tra l'altro, si osserva la presenza di stilature, vale a dire di incisioni praticate sulla malta fresca per "disegnare" in maniera regolare i giunti dell'apparecchio murario, come si osserva nelle consistenti tracce delle pareti del cortile.

Per contro, le pietre del conglomerato cementizio del nucleo sono piuttosto piccole $\left(100-400 \mathrm{~cm}^{2}\right.$ in prospetto) annegate in abbondante malta. La densità delle pietre in alcuni punti è elevata, a scapito della quantità di malta che le unisce. La disposizione irregolare del materiale lapideo denuncia poca attenzione alla creazione di una massa compatta e uniforme, così come attestato in molti cantieri fuori dall'Urbe, dove i nuclei in conglomerato non presentano una regolare stesura dei caementa per uniformi e compatti strati orizzontali. Si intuisce che alcune pietre più grandi furono disposte a mano, mentre le pietre più minute e la malta furono sommariamente gettate a colmare gli spazi tra le pietre più grandi. Ne consegue una presenza di vuoti, che non inficiano la capacità portante delle pareti solo grazie al notevole spessore murario e alla qualità della malta. Quest'ultima, non sempre abbondante, ha un'ottima qualità coesiva, evidente nello stato conservazione dei nuclei che hanno perduto i paramenti, dove la coerenza e consistenza della muratura è eccellente.

\footnotetext{
12 Con questo termine intendo indicare elementi lapidei che dopo essere stati cavati e ridotti eventualmente in elementi di dimensione più piccola, non vendono ulteriormente straformati in cantiere.
} 


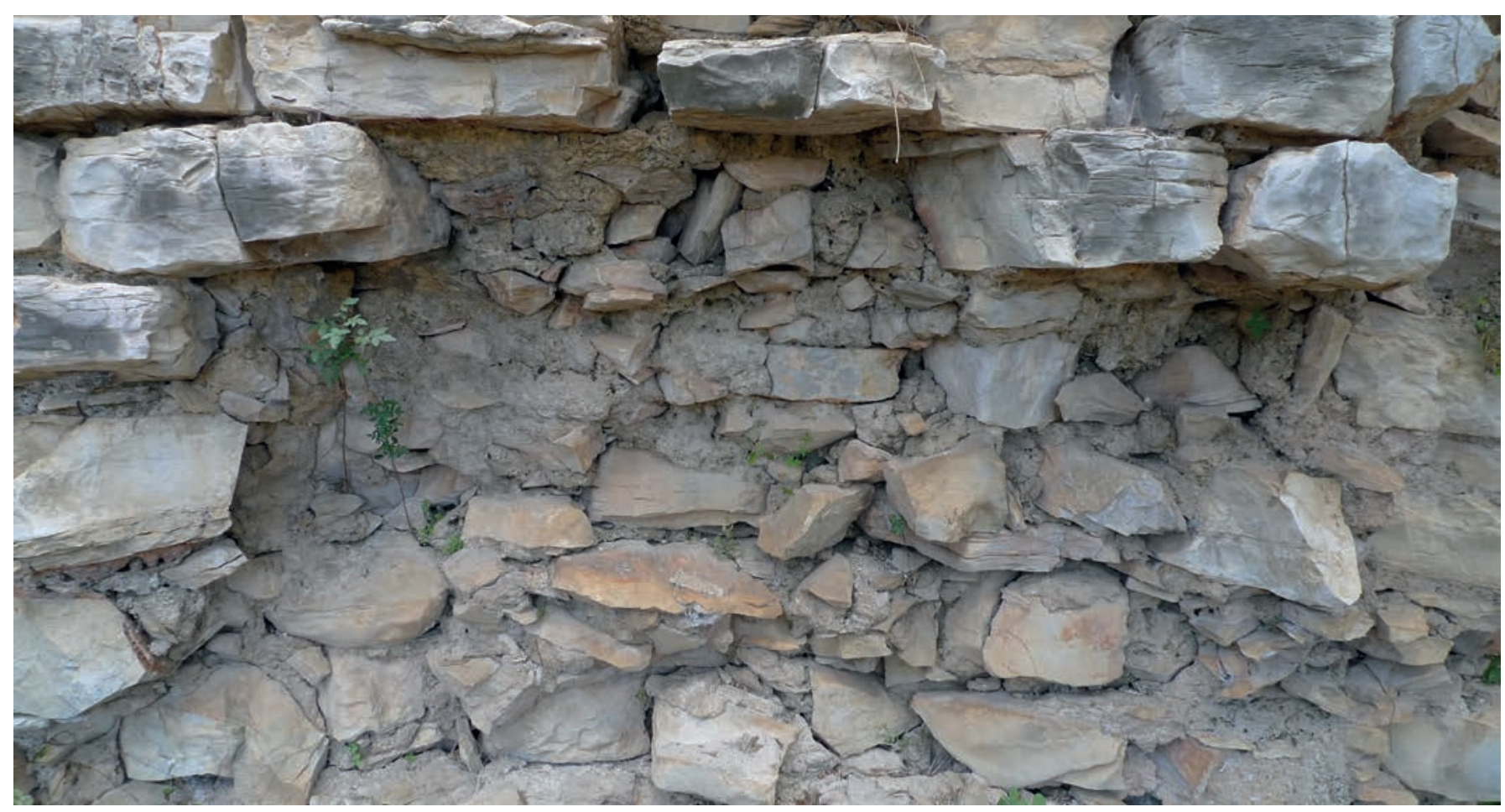

Fig. 10: Particolare del nucleo della parete Nord dell'ambiente (C).

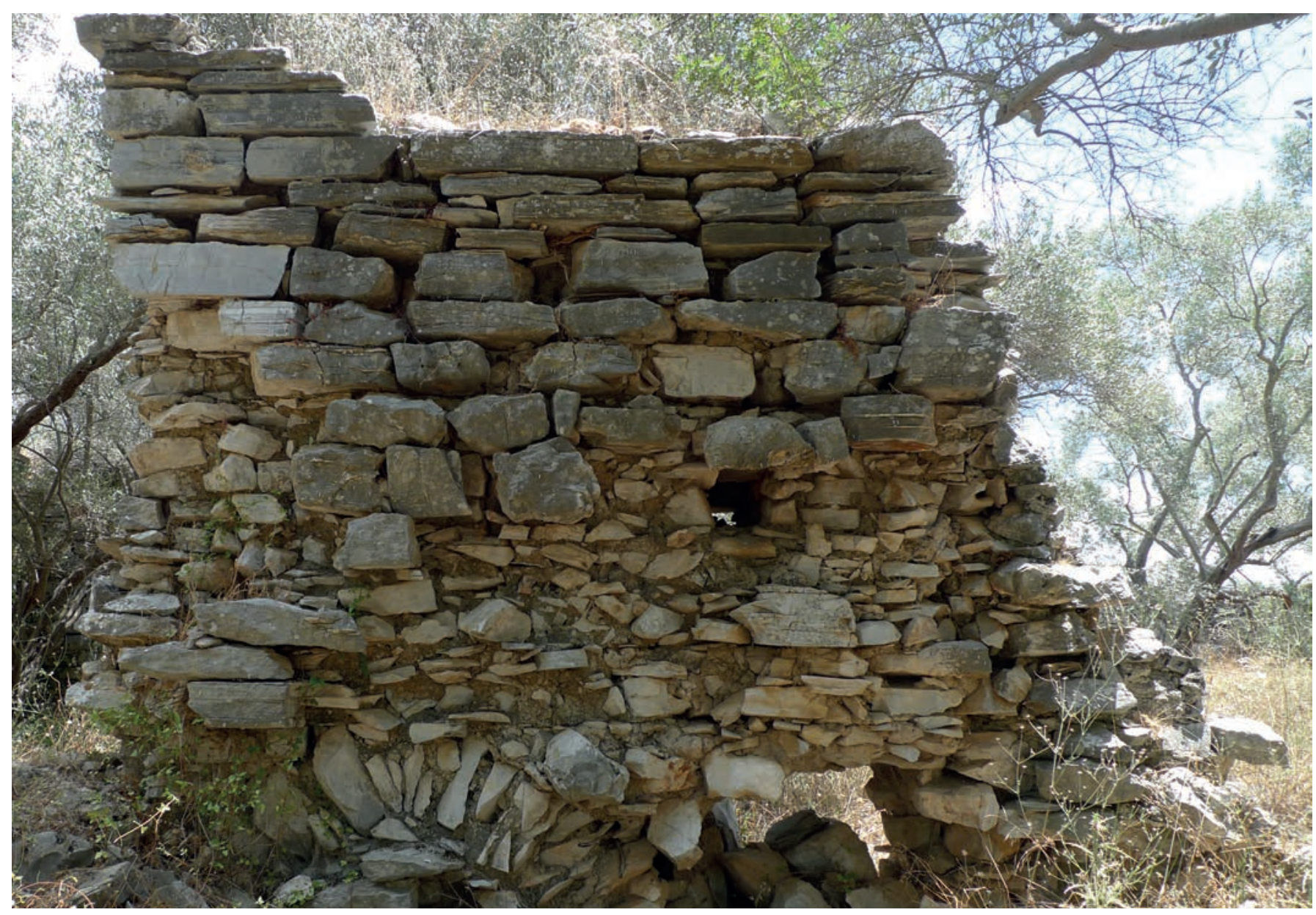

Fig. 11: Particolare del paramento della parete Est dell'ambiente (C). 
Vere e proprie legature tra il paramento e il nucleo, così come elementi diatoni, sono assenti. Le pietre dei paramenti, profonde ca. $30-40 \mathrm{~cm}$, sono a volte disposte "di testa", vale a dire con il lato lungo rivolto verso il nucleo, così da stabilire un collegamento strutturale con il nucleo, che nella parete $\mathrm{N}$ dell'ambiente (C) rimane minimo e poco profondo, così che che l'ammorsatura al nucleo rimane insufficiente. Si nota, comunque, come nel punto di imposta della volta le pietre che vanno a formare l'intradosso, che permangono orizzontali, hanno una profondità minimamente maggiore, tanto da non essere crollate con il paramento sottostante (Fig. 7).

Ricordiamo che Vitruvio a proposito delle murature romane costruite con un nucleo e due paramenti aveva esplicitamente sottolineato come tale sistema generasse una struttura tripartita ("tres suscitantur in ea structura crustae, due frontium et una media farturae"13). Per contro informava i suoi lettori che i Greci avevano la sana abitudine di costruire muri in pietra con blocchi diatoni che attraversavano la muratura da parete a parete così da legare la muratura in un unico corpo ${ }^{14}$.

Il motivo per cui Vitruvio prediligeva i sistemi costruttivi adottati nella Grecia pre-romana è da ricercarsi nel fatto che quello italico, così come maturato nel II-I secolo a.C., benché fosse più veloce, era più soggetto a dissesti. L'esperienza evidentemente insegnava che le murature tripartite erano spesso soggette al distacco del paramento dal nucleo, dovuto ad una differente rigidezza tra le parti, che poteva risultare in un diverso comportamento alle sollecitazioni e ai carichi. Il problema era divenuto cruciale nel cantiere di età imperiale, post-vitruviano, quando gli architetti di Roma si trovarono a risolvere problematiche strutturali sempre più impegnative. La risposta ai problemi evidenziati da Vitruvio prese la forma di una attenta selezione, lavorazione dei materiali utilizzati nel cantiere edile, che avrebbe condotto ad una sempre maggiore qualità costruttiva, percepibile nelle fabbriche di epoca imperiale, che spesso confluì in una maggiore attenzione alle legature tra nucleo e paramento e che è testimoniata da differenziate declinazioni regionali.

L'analisi della prassi costruttiva delle maestranze di Iasos può avvalersi di una serie di strutture di età imperiale, in cui emerge una consolidata applicazione di sistemi costruttivi romani, adattati al contesto locale (Fig. 12). L'impiego di materiale più minuto nei nuclei

\footnotetext{
13 Vitr. 2.8,7.

14 "Praetera interponunt singulos crassitudine perpetua utraque parte frontatos, quos ठıатоvouৎ appellant, qui maxime religando confirmant parietum
} soliditatem". Ibidem. murari, sembrerebbe in effetti rispondere alla logica della costruzione cementizia, in cui alla qualità della malta è associato l'impiego di pietre di piccola dimensione. Non si riscontra, tuttavia, una disposizione per corsi orizzontali delle pietre del nucleo, ma piuttosto il ricorso della tecnica "a sacco", con riempimento del nucleo dopo aver alzato il paramento per alcuni filari. Il materiale lapideo impiegato nei nuclei, non sempre omogeneo, sembra condizionato da una modesta selezione e rilavorazione del materiale, che, così come ricavato dai giacimenti rocciosi che affiorano a banchi distaccandosi secondo le faglie naturali, viene minimamente lavorato prima di essere messo in opera ${ }^{15}$. Le

\footnotetext{
15 A Iasos è largamente diffuso un cipollino scistoso di provenienza metamorfica che si altera a banchi poco compatti, da cui sono appunto ricavate le pietre per la realizzazione delle murature.
}

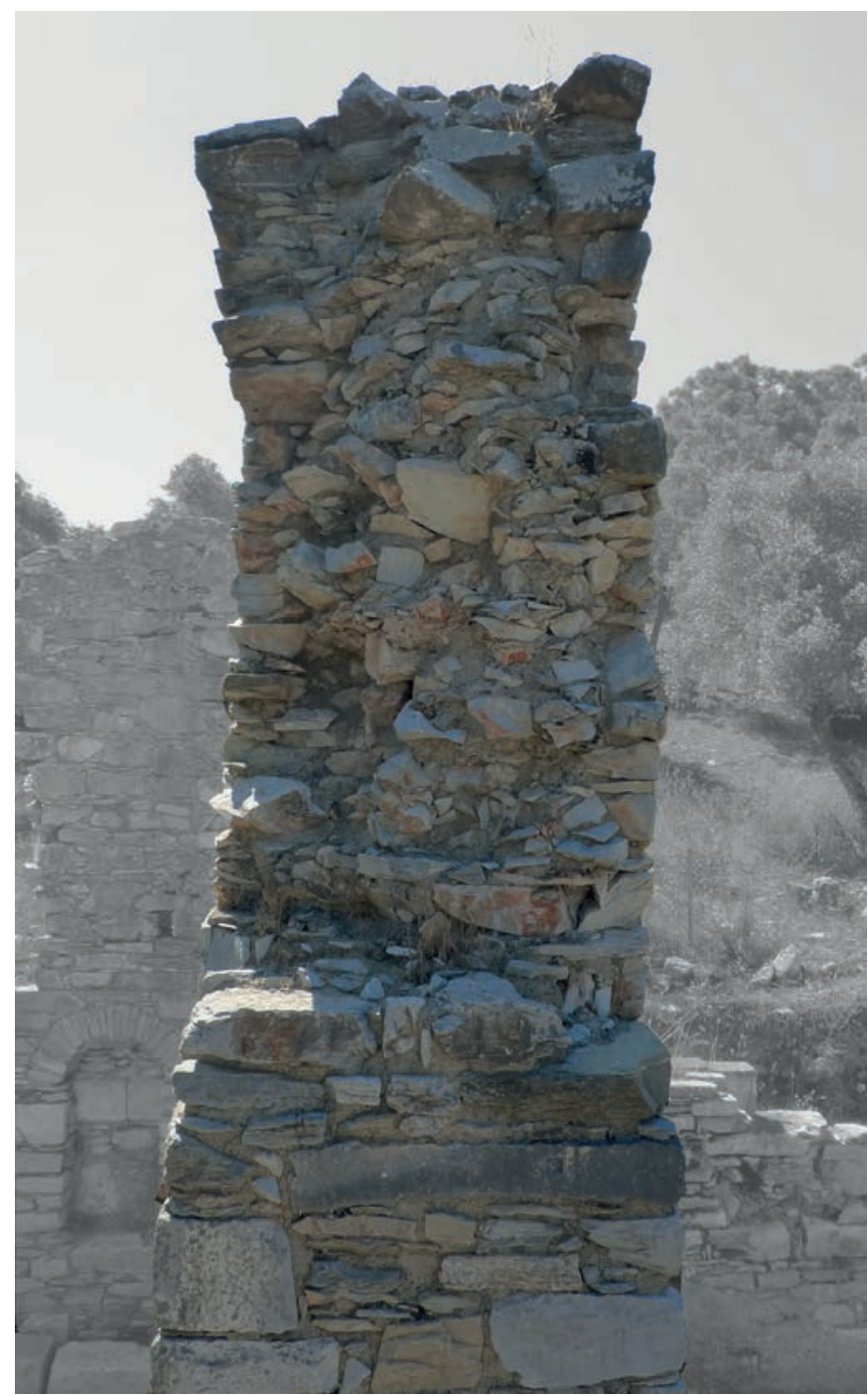

Fig. 12: Sezione di un muro in conglomerato del Caesareum nell'agorà. Il nucelo è composto da materiale mediamente di pezzatura piccola e sporadici blocchi più grandi disposti "a sacco". Il paramento è composto da pietre di media pezzatura. 
pietre più grandi sono destinate prevalentemente ai paramenti, quelle più piccole ai nuclei. Il processo costruttivo è pratico, per il fatto che sfrutta al meglio le risorse del territorio, confidando nella qualità della malta che viene prodotta. Si nota generalmente una buona tenuta dei paramenti ai nuclei murari, ottenuta con legature garantite da pietre poste "di testa", che, negli edifici più rappresentativi, come il cd. bouleuterion che affaccia sull'agorà, eseguito con paramenti in opera quadrata, risulta più evidente, grazie ai grandi conci sporadicamente legati al nucleo (Fig. 13). Qui la ripresa dei "getti" del nucleo in corrispondenza di ogni filare di paramento è particolarmente evidente.

La costruzione delle terme, pertanto, si inserisce nella tradizione locale, e, in particolare, in quella tipologia di murature in cui la dimensione delle pietre impiegate nei paramenti sembra prediligere un taglio piuttosto grande, mentre il nucleo è prevalentemente composto da materiale di minore dimensione, non omogeneo e non apparecchiato per $\operatorname{strati}^{16}$. A giudicare dalle creste delle murature ancora in piedi negli ambienti non riscaldati, sembra che l'ammorsatura tra paramento e nucleo sia qui più curata che non negli ambienti riscaldati $(\mathrm{C})$ e $(\mathrm{T})$. Ciò potrebbe spiegare il peggior stato di conservazione dei paramenti di quest'ultimi. L'assenza di cura nelle legature fra paramento e nucleo, di cui non si è qui in grado di offrire una interpretazione -e che probabilmente potrà essere risolta solo con lo scavo delle terme- è andata a condizionare pesantemente il comportamento meccanico di questi in concomitanza di sollecitazioni che anziché distribuirsi omogeneamente nella sezione muraria, si concentrano nella spessa e

\footnotetext{
16 Fanno eccezione alcune murature in cui il nucleo è composto da pietre di maggiore dimensione. Un'analisi più dettagliata e puntuale di tutte le murature delle terme esula dai fini del presente scritto.
}

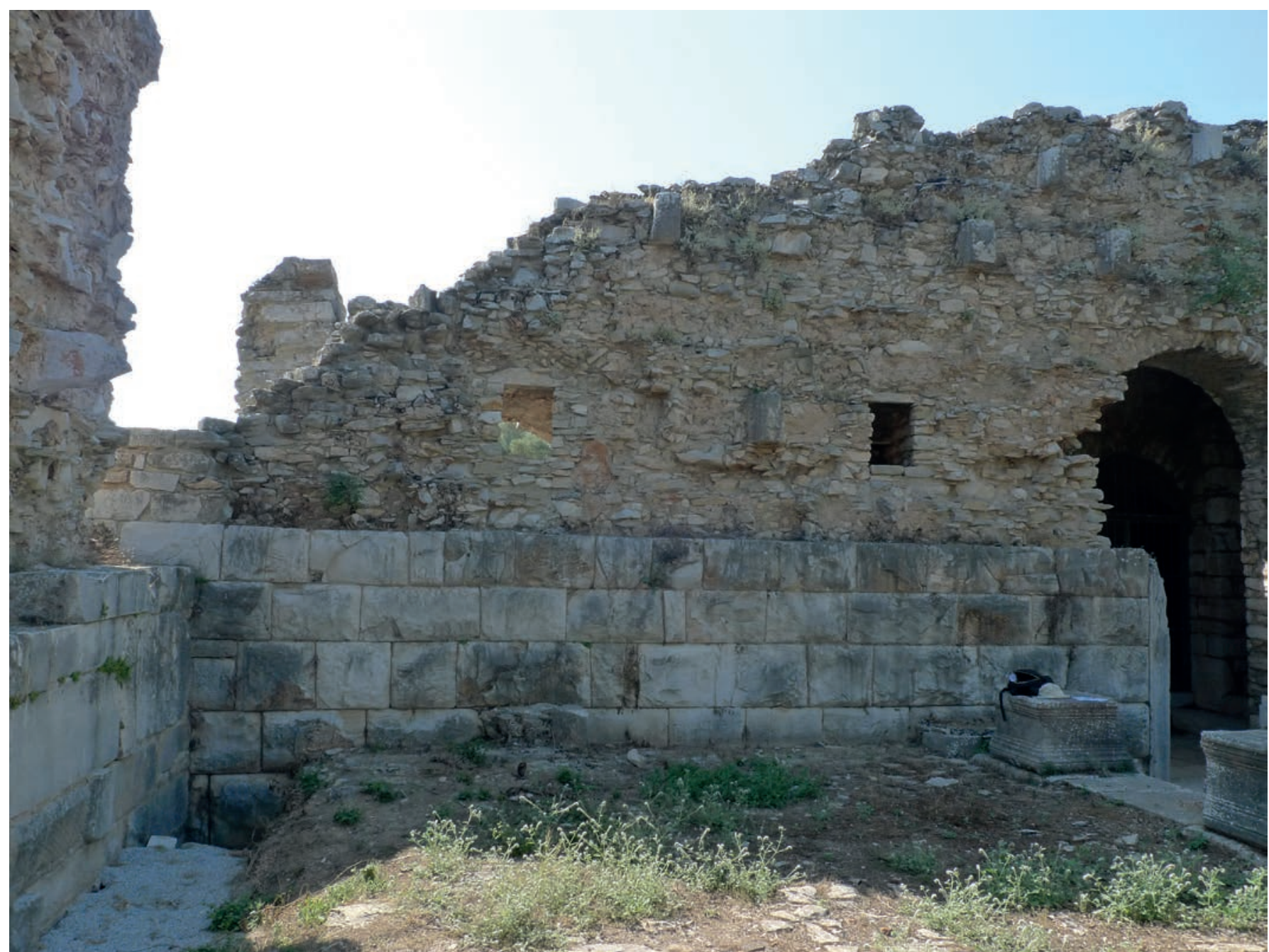

Fig. 13: Prospetto Ovest del Bouleuterion. Nella parte in cui blocchi in opera quadrata del paramento sono stati asportati si distinguono blocchi di testa destinati ad accrescere l'ammorsatura tra nucleo e paramento e l'altezza dei getti di conglomerato, corrispondente con l'altezza dei blocchi del paramento. 
rigida pelle. Quest'ultima proprio per la sua attitudine a deformarsi in misura minore ai carichi, dovuta alla scarsa quantità di malta tra una pietra e l'altra, tende ad assorbire le forze che investono la muratura in maniera superiore rispetto al nucleo. In assenza di opportune legature ciò si traduce in un distacco ed, eventualmente, nel crollo del paramento. Da quanto esposto, sembrerebbe che la parete Nord dell' ambiente (C) proprio per il suo spessore eccezionale, la maggiore altezza e le scarse ammorsature sia stata investita da un problema che ha causato una forte instabilità delle pietre del paramento, senza investire il paramento sul lato prospicente il frigidarium (F2).

\section{Archi e volte}

A Iasos genericamente archi e volte sono in pietra, con qualche eccezione, come, ad esempio, nelle terme del porto interno (Fig. 1), dove furono impiegati archi e piattabande integralmente in laterizio. Volte ancora integre sono visibili nei mausolei che affacciano sul porto commerciale (Fig. 14). Qui il primo tratto dall'imposta alle reni è realizzato con pietre disposte per filari orizzontali, mentre il tratto superiore è eseguito con lastre di scisto apparecchiate radialmente e unite da sottili strati di malta. La centina fu impiegata solo per la costruzione del tratto con pietre disposte radialmente, come testimoniano i fori lasciati dalle travi che la sorreggevano, collocati all'altezza delle reni. Soluzione che ottimizzava legname e lavorazioni necessarie alla costruzione della centina, ridotta ad un settore minore rispetto al pieno sesto della volta. Nel contempo, essa riduceva la luce che dovevano coprire le travi che sostenevano il peso della centina e della volta sovrastante. Di fatto simili "centine volanti", evitavano la costruzione di armature lignee che scaricavano il peso della centina e della volta

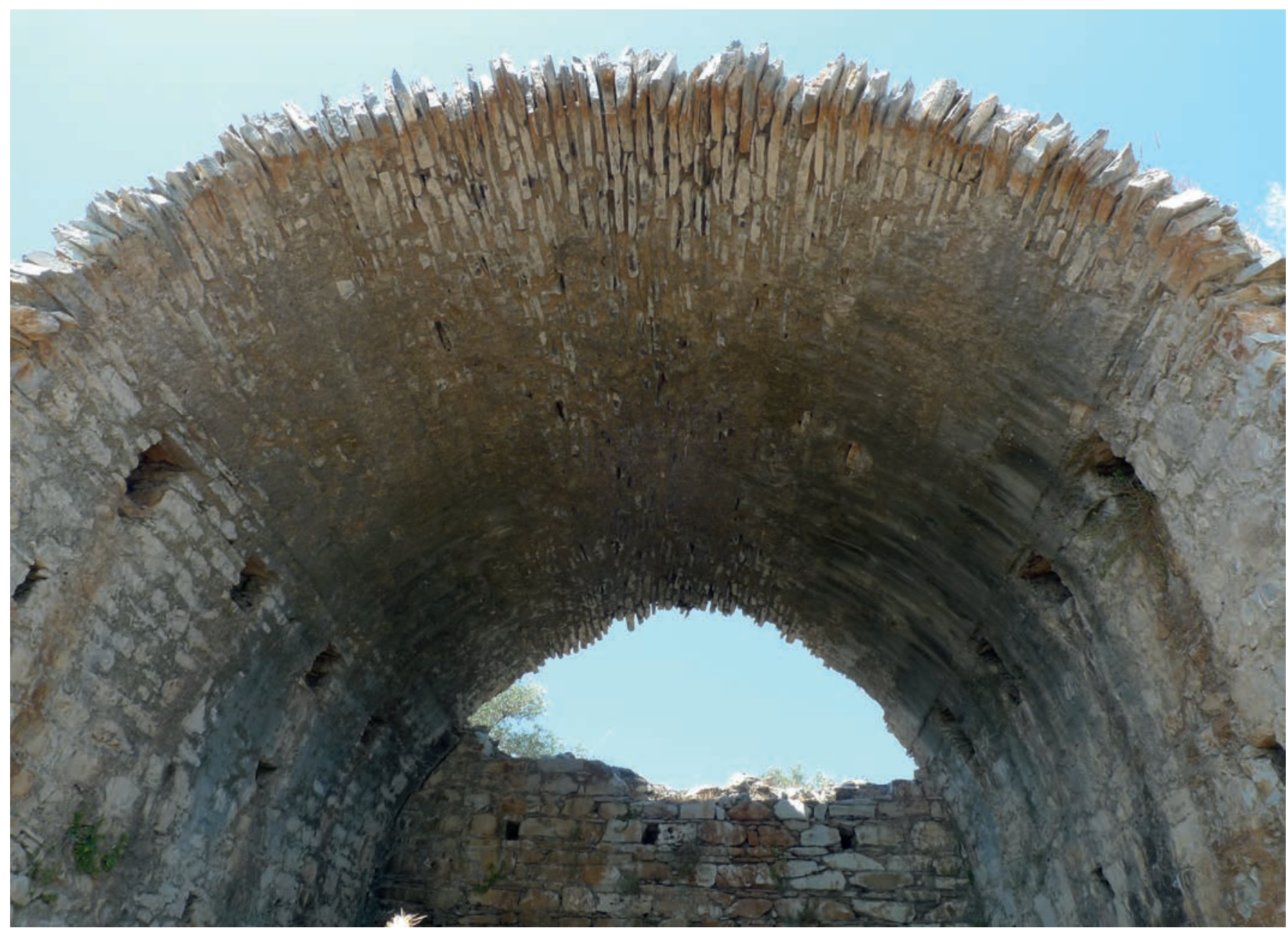

Fig. 14: Veduta della volta a botte di un mausoleo di Iasos. 
in costruzione direttamente sul terreno. Il processo costruttivo così concepito risultava efficace dal punto di vista cantieristico, per il fatto che nella parte più alta della struttura venivano sollevate pietre più leggere. Ma era anche di elevata resistenza, in quanto la parte inferiore, realizzata in continuità con la parete, andava a realizzare un corpo unico e compatto tra il piedritto e il primo tratto della volta che era un tutt'uno con il rinfianco. Al di sopra di esso era la volta estradossata, vale a dire con estradosso curvo, di spessore pari alle lastre di scisto.

Nelle grandi terme le volte sono crollate; si conservano solo alcuni tratti che raggiungono l'altezza delle reni. Il sistema delle "centine volanti" sembra applicato nella maggior parte degli ambienti, ad eccezione del frigidarium (F1 e F2). Le travi che sostenevano la centina erano inserite all'imposta e non all'altezza delle reni. La sezione schematica (Fig. 15) della parete del caldarium (C) e dell'attiguo frigidarium (Fig. 2: F2) aiuta a inquadrare meglio la costruzione.

La volta di (C) era ampia ca. 8,60 m. Quella del frigidarium $6,20 \mathrm{~m}$. In entrambe le pietre sono disposte per filari orizzontali fino alle reni. La qualità e compattezza della costruzione è ben visibile dal lato del frigidarium (F1) (Fig. 16), dove sia il paramento che la volta presentano uno stato di conservazione ancora elevato. La parte della volta crollata doveva essere eseguita con pietre radiali, come nel tratto di volta conservato nell'apodyterium (Fig. 15: sez. Y-Y). Una serie di aperture, poi tamponate (Fig. 16: a), che si aprivano nella volta del frigidarium (F1 e F2), necessarie ad illuminare gli ambienti, altrimenti bui, sembrano confermare un profilo estradossato sia per la volta del caldarium che del frigidarium, così come attestato nel mausoleo sopra descritto.

Quanto osservato rende improbabile l'ipotesi che il dissesto del paramento sia stato dovuto alle tensioni trasmesse dalle volte sul muro sottostante. A vista non si notano infatti deformazioni o fuori piombo che possano essere stati causa di dissesti o crolli. Tanto più che la spinta della volta dell'ambinete (C) era equilibrata da quella della volta di (F1), così da annullarsi a vicenda. La stessa apertura della grande breccia a fianco della nicchia così come le due nicchie sulla contro-parete (aperte in un secondo momento. V. Fig. 16) non sembrano avere causato lesioni sulle porzioni murarie sovrastanti. In mancanza di dati che possano meglio spiegare i motivi per cui le volte crollarono, è prudente soffermarsi alla sola constatazione che il loro crollo non turbò la solidità della parete portante con la nicchia e tantomeno può aver causato il dissesto del paramento verso l'ambiente (C).

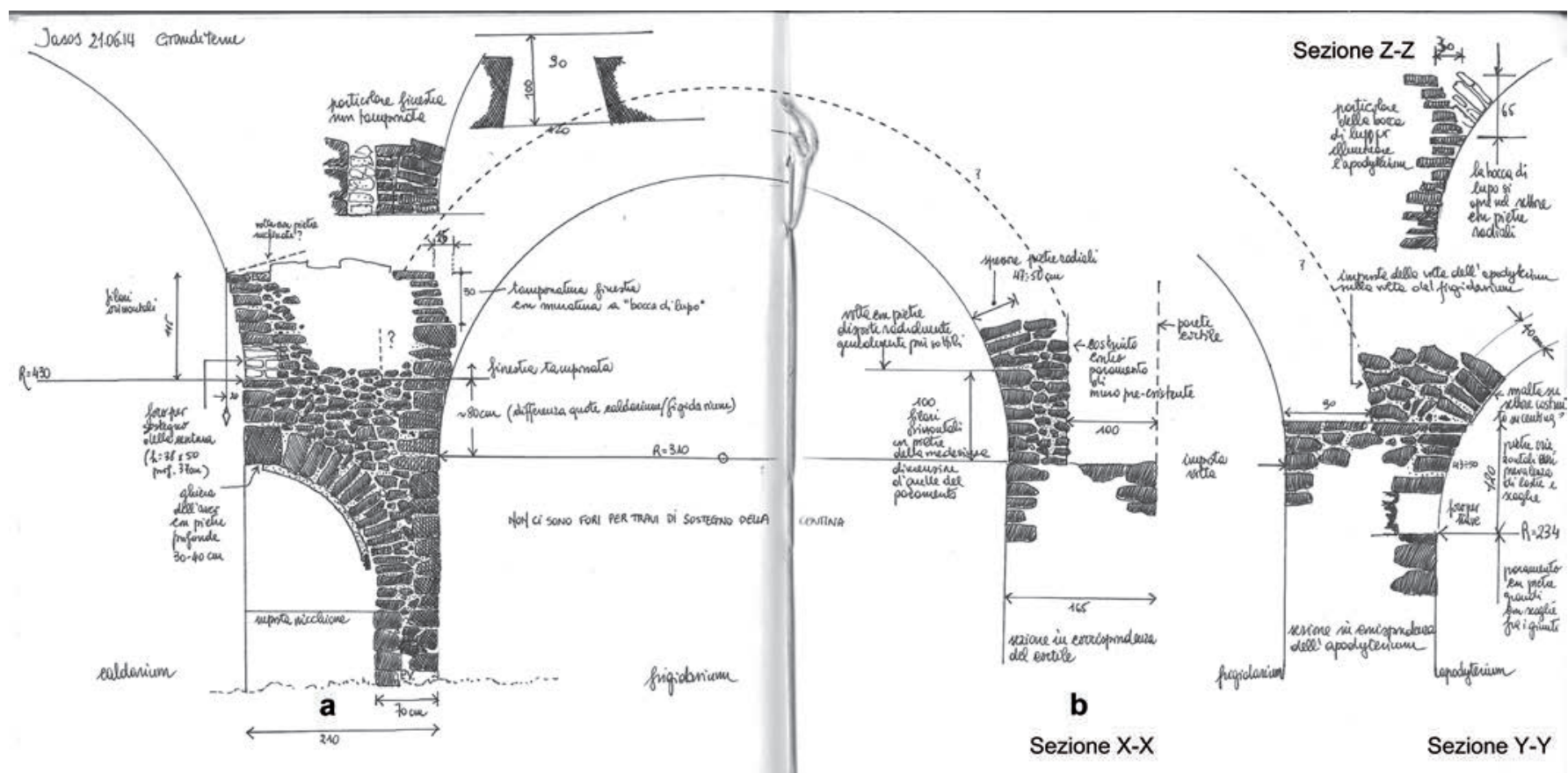

Fig. 15: Sezioni diagrammatica con indicazione delle tessiture murarie (per ubicazione delle linee di sezione v. fig. 2). 


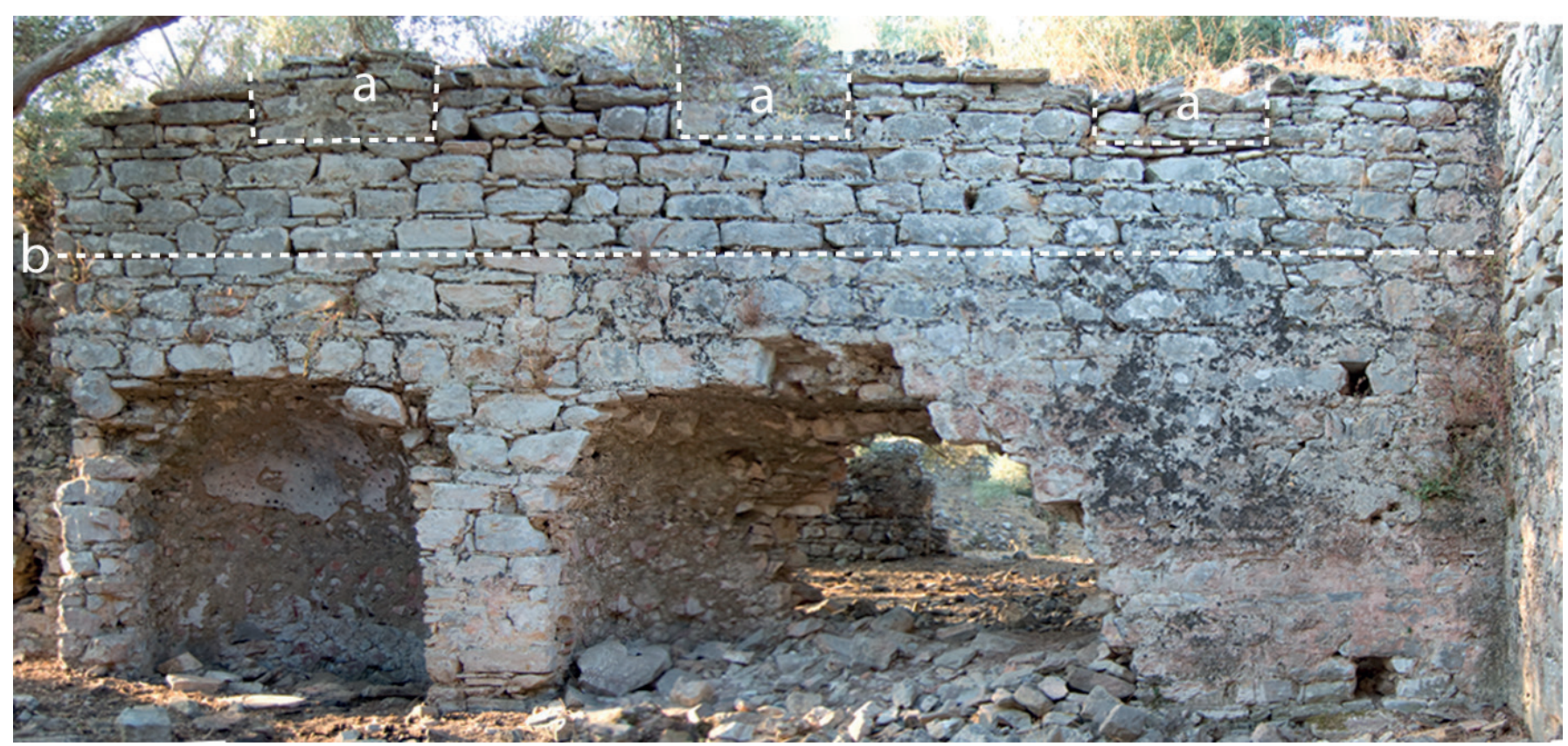

Fig. 16: Veduta della contro-parete del muro Nord dell'ambiente C (muro Sud dell'ambiente F). La grande lacuna al centro corrisponde alla nicchia al centro della parete di (C). a) finestre successivamente tamponate; b) imposta della volta a botte di (F). Si noti la differente dimensione delle pietre di (F) rispetto a quelle di (C).

\section{CONSIDERAZIONI CONCLUSIVE}

Le differenze fra i paramenti verso l'ambiente (C) e verso (F1) porterebbero a ipotizzare un intervento avvenuto specificatamente sulla parete del caldarium, dove i giunti tra le pietre del paramento dovevano essere in origine intercalate da numerose scaglie di pietra utili a colmare i giunti irregolari fra le pietre, così come si osserva nella parete del frigidarium, dove tra l'altro è abbondante la malta nei giunti tra una pietra e l'altra (Fig. 16). La presenza delle zeppe metalliche, attorno alle quali vi è malta, denuncia un intervento localizzato e specifico pensato solo per le pareti del caldarium dove la malta fu evidentemente inserita in un secondo tempo all'infissione degli elementi metallici, in giunti in cui mancavano anche zeppe di pietra. Le inzeppature ristabilivano così uno stato di compressione tra le pietre, per qualche motivo venuto meno.

L'ipotesi che si può formulare è che il muro a cui era applicata l'intercapedine per il riscaldamento delle pareti dell'ambiente caldo, investito da un sisma, abbia subito un dissesto della pesante fodera/paramento del nucleo murario. Il danno causò probabili fessurazioni sulla contro-parete riscaldata, con fuoriuscita dei fumi provenienti dall'ipocausto. L'intercapedine fu pertanto rimossa, mettendo in luce una diffusa instabilità delle pietre, che, per effetto della diversa rigidezza tra il nucleo e il paramento ${ }^{17}$ e per l'esiguità delle legature, si erano mosse, senza però distaccarsi dalla parete. A questo punto la malta e le zeppe lapidee furono rimosse dai giunti, per consentire l'inserimento dei cunei e zeppe di ferro. La soluzione, se è corretta la nostra interpretazione, consentiva di rimettere in carico le pietre del paramento, senza dovere provvedere allo smontaggio e ricostruzione dell'intero paramento, operazione particolarmente complessa in presenza di una volta poggiante in parte sul paramento. È inoltre plausibile che la scelta dell'inusuale intervento di consolidamento fosse stata condizionata dal fatto che il danno si era materializzato solo su una parete delle terme.

Esempi di simili consolidamenti sono piuttosto rari e poco noti nella letteratura tecnica. Penso che il dissesto di Iasos possa essere confrontato con due altri esempi.

Un primo possibile confronto è quello di un arco di scarico nella Yedi Kule a Istanbul, dove numerosi chiodi furono inseriti tra i laterizi, plausibilmente per mettere a contrasto i laterizi nei punti dove si erano aperte delle fessure (Fig. 17). Veri e propri cunei di ferro come quelli presenti nell'arco della grande nicchia delle terme di Iasos, dove, similmente, i giunti vennero successivamente riempiti di malta.

7 La minore quantità di malta tra le pietre, rispetto a quella del conglomerato del nucleo, giustifica una maggiore rigidezza e, pertanto, una tendenza ad assorbire in misura maggiore le tensioni dinamiche generate dal sisma. 


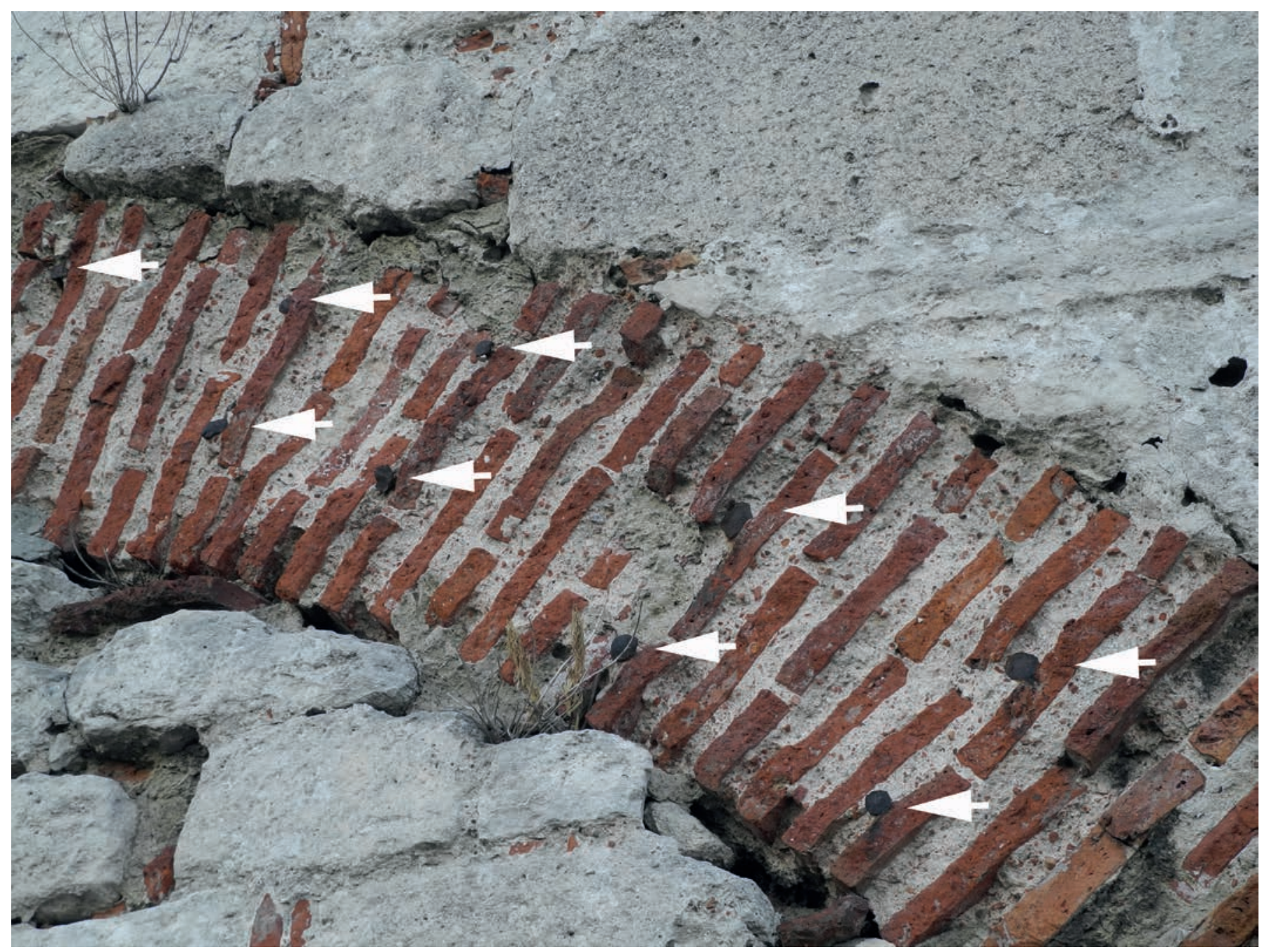

Fig. 17: Istanbul, Yedi Kule. Chiodature di ferro nei giunti di un arco laterizio (fotografia C. M. Amici).

Un secondo esempio si trova a Rodi. L'angolo sul mare della grande torre di Naillac nel porto di Rodi (Fig. 18), eseguito nella parte inferiore, a contatto con il mare, con blocchi di reimpiego di marmo legati con grappe di ferro, per effetto delle onde marine, si era dissestato. I blocchi allentati nella loro coesione originaria, trovandosi sotto alla poderosa torre (oggi $\mathrm{crollata}^{18}$ ) e in posizione critica per l'esecuzione di uno smontaggio e rimontaggio della parte dissestata, fu consolidata con zeppe di ferro, alcune delle quali ancora visibili (Fig. 19). Anche in questo caso la soluzione deve essere stata considerata come la più vantaggiosa, considerata

\footnotetext{
18 V. Manoussou-Ntella 2010.
}

la difficoltà di un intervento dall'esterno della torre, in mare aperto e dovendo per forza smontare una porzione consistente di muratura in opera quadrata, per poi rimontarla e rimetterla in carico.

L'esiguità di esempi conosciuti è probabilmente legata allo scarso interesse che in passato hanno avuto interventi di consolidamento, spesso passati inosservati o semplicemente poco compresi. Certamente l'intervento di Iasos lascia aperti alcuni dubbi, come ad esempio la totale assenza di danno sulla parete di (F1), laddove l'accelerazione provocata dal sisma sui carichi verticali avrebbe dovuto ripercuotersi anche sul paramento opposto a quello dissestato. Ma non sempre è possibile ricostruire tutti gli eventi incorsi nella storia di un manufatto e, di conseguenza, capire le 


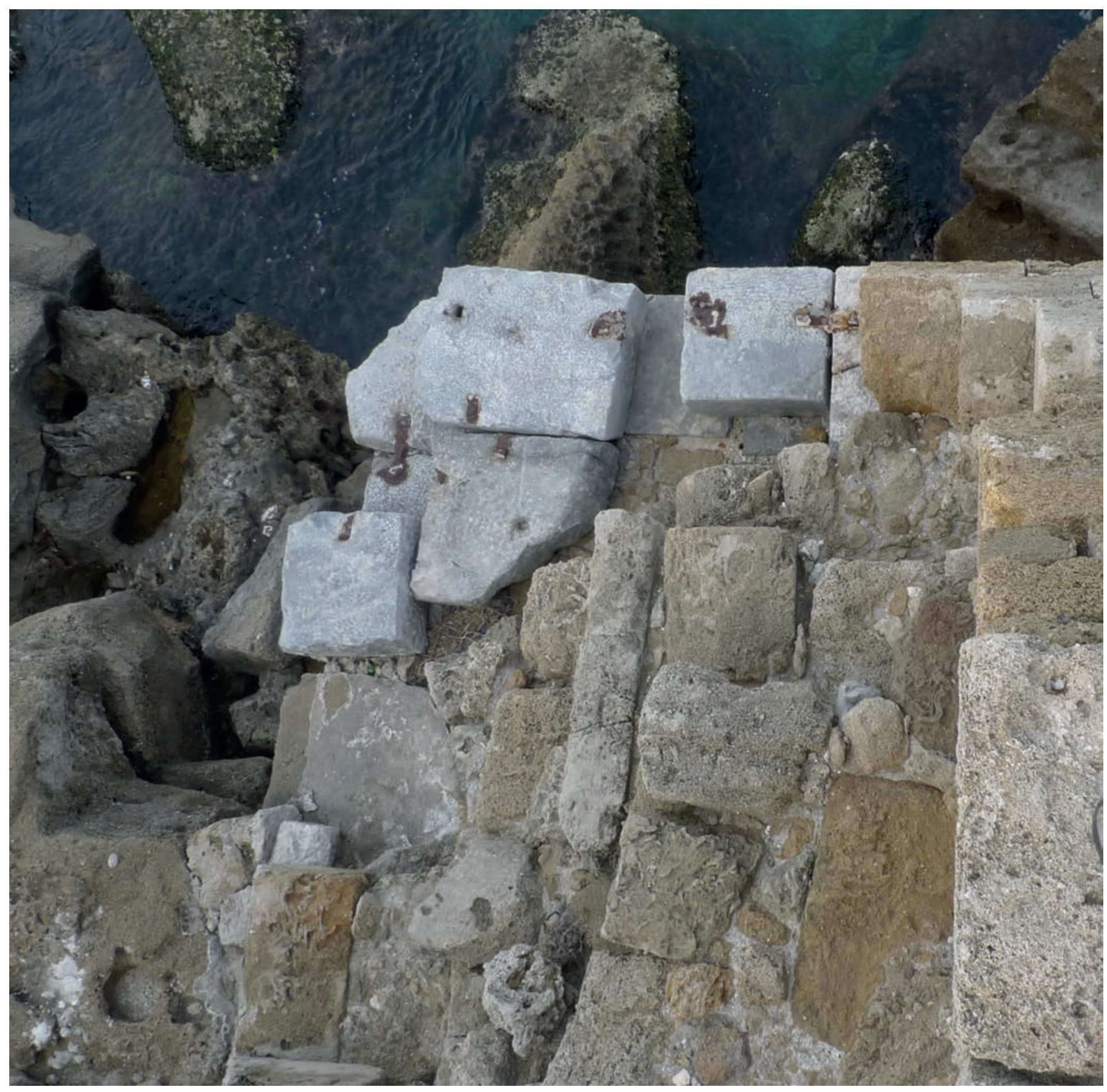

Fig. 18: Rodi, torre di Naillac. Veduta dall'alto dell'angolo dissestato in un angolo della torre, esposto alle onde marine.

dinamiche che hanno portato le strutture ad assumere le caratteristiche che noi oggi osserviamo. Le interpretazioni possibili di una realtà complessa come quella di un rudere sono molteplici e occorre sapersi fermare ai dati suggeriti dall'evidenza e non formulare ipotesi concatenanti difficilmente verificabili. Nel caso delle terme di Iasos c'è da sperare che lo scavo, quando verrà eseguito, potrà sciogliere $\mathrm{i}$ diversi nodi interpretativi rimasti aperti. 


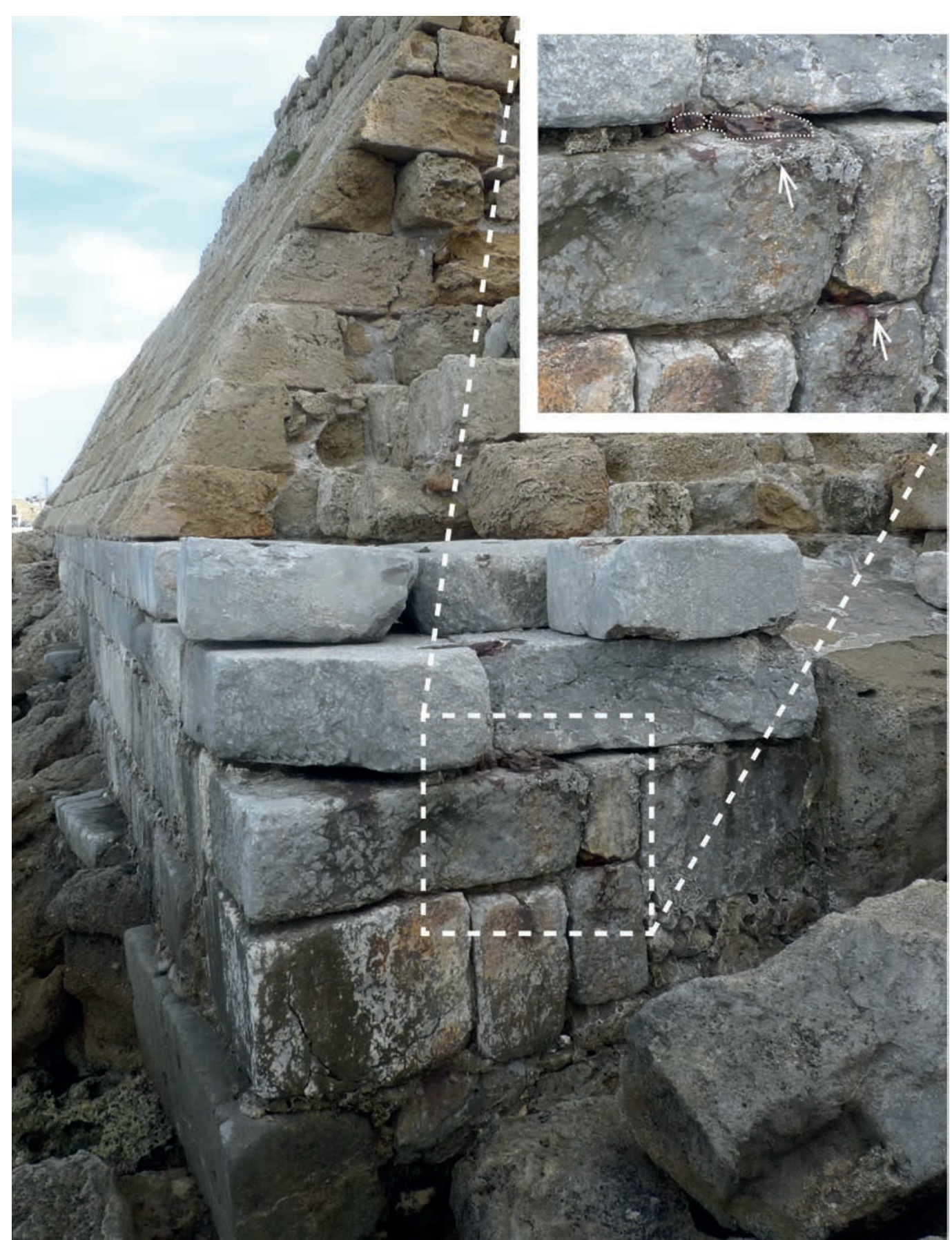

Fig. 19: Rodi, torre di Naillac. Particolare di cunei metalliche tra i blocchi di cui alla Fig. 18.

\section{RINGRAZIAMENTI}

Questo articolo è il frutto del generoso invito di Alessandro Viscogliosi a recarmi a Iasos nel mese di giugno 2014. In tale occasione mi soffermai a lungo sulla parete qui descritta. Il presente scritto, sollecitato da A. Viscogliosi e basato sulla condivisione di molte sue osservazioni tecniche, è ora, grazie alla disponibilità del medesimo, oggetto di questa pubblicazione. Si ringrazia Alessio La Paglia, che ha elaborato i rilievi e lo studio delle terme, per il materiale e le informazioni che ha voluto generosamente darmi. Sono grato a Carla Maria Amici per le discussioni e le numerose osservazioni nonché per avermi segnalato l'intervento sull'arco della Yedi Kule e fornito l'immagine qui pubblicata. Le stimolanti osservazioni dei due anonimi referees hanno contribuito positivamente alla stesura finale dell'articolo. Ringrazio R. Perucchio per le discussioni sulle problematiche di carattere strutturale. 


\section{BIBLIOGRAFÍA}

Bianchini, M. e Vitti, M. 2017: I Mercati di Traiano. L'Erma di Bretschneider, Roma.

Giuliani, C. 2006: L'edilizia nell'antichità. Carocci, Roma.

Hoffmann, A. 2009: Die Casa del Fauno in Pompeji (VI 12). Reichert, Wiesbaden.

Manoussou-Ntella, K. 2010: "Le paysage culturel et les monuments symboles disparus de la ville de Rhodes", Europa Nostra bulletin scientifique, 64, pp. 59-74.
Viscogliosi, A. 2009: “Il 'castello di Terraferma' a Iasos", Iasos di Caria, 15 , pp. 6-13.

Viscogliosi, A. 2010: "Il 'castello di Terraferma' a Iasos. Indagini 2009", Iasos di Caria, 16, pp. 19-26.

Yegül, F. 1992: Baths and Bathing in Classical Antiquity. MIT Press, Cambridge. 